\title{
On the Convergence of a Non-linear Ensemble Kalman Smoother
}

\author{
El Houcine Bergou* $\quad$ Serge Gratton ${ }^{\dagger} \quad J^{*}$ Mandel $^{\ddagger}$
}

\begin{abstract}
Ensemble methods, such as the ensemble Kalman filter (EnKF), the local ensemble transform Kalman filter (LETKF), and the ensemble Kalman smoother (EnKS) are widely used in sequential data assimilation, where state vectors are of huge dimension. Little is known, however, about the asymptotic behavior of ensemble methods. In this paper, we prove convergence in $L^{p}$ of ensemble Kalman smoother to the Kalman smoother in the large-ensemble limit, as well as the convergence of EnKS-4DVAR, which is a Levenberg-Marquardt-like algorithm with EnKS as the linear solver, to the classical Levenberg-Marquardt algorithm in which the linearized problem is solved exactly.
\end{abstract}

\section{Introduction}

Data assimilation is the process of blending estimates of a given system state, in the form of observational information and a prior knowledge [18]. The Kalman filter/smoother (KF/KS) $[6,8,14]$ and the three and four-dimensional variational assimilation system (3DVAR/4DVAR) [7,31] are among well-known algorithms used in data assimilation. Kalman filters estimate the state sequentially by seeking an analysis that minimizes the posterior variance, while the 3DVAR and 4DVAR methods produce posterior maximum likelihood solutions through minimization of an objective function. For high-dimensional problems, the ensemble Kalman filter/smoother (EnKF/EnKS) [16, 8] and their variants have been proposed as Monte Carlo derivative-free alternatives to the KF and KS, with the intractable state covariance in the KF or in the KS replaced by the sample covariance computed from an ensemble of realizations.

The purpose of this paper is to provide theoretical results for the method originally proposed in [25], called EnKS-4DVAR. The EnKS-4DVAR method uses an ensemble Kalman smoother as a linear solver in the Gauss-Newton or Levenberg-Marquardt method to minimize the weak-constraint 4DVAR objective function. Further details on implementation and computational results can be found in [25].

The equivalence of the Kalman smoother and incremental variational data assimilation has been known for a long time; see, e.g., [2, 21]. Hybridization of variational and ensemble-based methods has been a topic of interest among researchers in recent years [11, 35, 34, 29, 4, 5]. The maximum

*MaIAGE, INRA, Université Paris-Saclay, 78350 Jouy-en-Josas, France, and King Abdullah University of Science and Technology (KAUST), Thuwal, Saudi Arabia.

${ }^{\dagger}$ INP-ENSEEIHT and CERFACS, Toulouse, France.

${ }^{\ddagger}$ University of Colorado Denver, Denver, CO, USA, and Institute of Computer Science, Academy of Sciences of the Czech Republic, Prague, Czech Republic. Partially supported by the U.S. National Science Foundation under the grant DMS-1216481, the Czech Science Foundation under the grant 13-34856S and the Fondation STAE project ADTAO. 
ensemble likelihood filter (MELF) [35] uses repeated EnKF on the tangent problem to minimize the objective function over the span of the ensemble. The iterated ensemble Kalman filer (IEnKF) [29] solves the Euler equations for the minimum by Newton's method, preconditioned by a square root ensemble Kalman filter, while [4] adds a regularization term, similar to the Levenberg-Marquardt method, and [5] extends the IEnK method to strong-constraint 4DVAR. The IEnKF uses a scaling of the ensemble, called the "bundle variant" to approximate the derivatives (tangent operators), achieving a similar effect as the use of finite differences here. The four-dimensional ensemble-based variational data assimilation (4DEnVar) of $[23,24,22]$ minimizes the 4DVAR objective function over the span of the ensemble.

Usually, in the formulation of the ensemble based methods (EnKF/EnKS and their variants), each ensemble member is considered as a vector in $\mathbf{R}^{n}$, that is, each vector is regarded as a sample point of a random vector. In this paper, we investigate a different way to interpret such algorithms, similarly as in [26, 19], namely, each ensemble member is considered as a random vector and not merely as vector of $\mathbf{R}^{n}$. In fact, the elements of the EnKF/EnKS can be seen as random vectors instead of their realizations. Surprisingly, in this case little is known about the asymptotic behavior of the EnKF/EnKS and other related ensemble methods. This is in contrast to particle filters, for which the asymptotic behavior as the number of particles increases to infinity is well studied. An important question related to EnKF/EnKS and related ensemble methods is a law of large numbers-type theorem as the size of the ensemble grows to infinity. In [26, 19], it was proved that the ensemble mean and covariance of EnKF converge to those of the KF, as the number of ensemble members grows to infinity, but the convergence results are not dimension independent. The analysis in [26] relies on the fact that ensemble members are exchangeable and uses the uniform integrability theorem, which does not provide convergence rates; in [19], stochastic inequalities for the random matrices and vectors are used to obtain the classical rate $1 / \sqrt{N}$, where $N$ is the ensemble size, but it relies on entry-by-entry arguments. Convergence in $L^{p}$ with the rate $1 / \sqrt{N}$ independent of dimension (including infinite) was obtained recently for the square root ensemble Kalman filter [17]. These analyses apply to each time step separately rather than for the long-time behavior. The EnKF was proved to be well-posed and to stay within a bounded distance from the truth, for a class of dynamical systems, with the whole state observed, and when a sufficiently large covariance inflation is used [15].

In this paper, we extend the convergence result of [26] to EnKS, and apply the extension to EnKS-4DVAR. The randomness of the elements of EnKS implies that, in contrast to the EnKS4DVAR algorithm presented in [25], the coefficients and the solution of the linearized subproblem at each iteration are random. We investigate also the asymptotic behavior of this algorithm. We show the convergence of the EnKS to the KS in $L^{p}$ for all $p \in[1, \infty)$ in the large ensemble limit, in the sense that the ensemble mean and covariance constructed by EnKS method converge to the mean and covariance of the KS respectively in $L^{p}$. Finally, we show the convergence of the EnKS-4DVAR iterates to their corresponding iterates in the classical Levenberg-Marquardt algorithm. Since the EnKS-4DVAR algorithm uses finite differences for approximating derivatives, (i) we start by showing the convergence in probability of its iterates to the iterates generated by the algorithm with exact derivatives as the finite differences parameter goes to zero, (ii) then we prove the convergence in $L^{p}$ of its iterates as the size of the ensemble grows to infinity.

The paper is organized as follows: in Section 2 we recall some definitions and preliminary results that will be useful throughout the paper. Section 3 introduces nonlinear data assimilation. Section 4 contains the statements of the $\mathrm{KF}$ and the EnKF, and recalls the convergence properties of the 
EnKF as the ensemble size increases to infinity. Section 5 gives statements of the KS and the EnKS, and extends the convergence properties of the EnKF as the ensemble size goes to infinity, to the EnKS. Finally, Section 6 recalls the EnKS-4DVAR algorithm and presents the convergence properties.

\section{Preliminaries}

We recall definition of sequence of random vectors exchangeability, the notion of convergence in probability and in $L^{p}$ of random elements. Then we present several lemmas, which will be useful for the following of the paper.

Definition 1 (Exchangeability of random vectors). A set of $N$ random vectors $\left[X^{1}, \ldots, X^{N}\right]$ is exchangeable if their joint distribution is invariant to a permutation of the indices; that is, for any permutation $\pi$ of the numbers $1, \ldots, N$ and any Borel set $B$,

$$
\mathbb{P}\left(\left[X^{\pi(1)}, \ldots, X^{\pi(N)}\right] \in B\right)=\mathbb{P}\left(\left[X^{1}, \ldots, X^{N}\right] \in B\right) .
$$

Clearly, an i.i.d sequence is exchangeable.

If $X$ is a random element (either vector or matrix), we use $|X|$ to denote the usual Euclidean norm (for vectors) or spectral norm (for a matrix). For $1 \leq p<\infty$, denote

$$
\|X\|_{p}=E\left(|X|^{p}\right)^{1 / p} .
$$

The space $L^{p}$ (of vectors or matrices) consists of all random elements $X$ (with values in the same space) such that the $E\left(|X|^{p}\right)<\infty$. Identifying random elements equal a.s., we have that $\|\cdot\|_{p}$ is a norm on the space $L^{p}$. Convergence in $L^{p}$ is defined as the convergence in this norm. Note that if the element $X$ is deterministic,

$$
\|X\|_{p}=E\left(|X|^{p}\right)^{1 / p}=\left(|X|^{p}\right)^{1 / p}=|X| .
$$

Definition 2 (Convergence in probability). A sequence $\left(X^{k}\right)$ of random vectors converges in probability towards the random vector $X$ if for all $\epsilon>0$,

$$
\lim _{k \rightarrow \infty} \mathbb{P}\left(\left|X^{k}-X\right| \geq \epsilon\right)=0
$$

i.e.,

$$
\forall \epsilon>0 \forall \tilde{\epsilon}>0 \exists k_{0} \forall k \geq k_{0}: \mathbb{P}\left[\left|X^{k}-X\right| \leq \epsilon\right] \geq 1-\tilde{\epsilon} .
$$

Convergence in probability will be denoted by

$$
X^{k} \stackrel{\mathrm{P}}{\rightarrow} X \text { as } k \rightarrow \infty .
$$

The concept of convergence in probability and the notation are extended in an obvious manner to the case when the random vectors are indexed by $\tau>0$. Then

$$
X^{\tau} \stackrel{\mathrm{P}}{\rightarrow} X \text { as } \tau \rightarrow 0
$$

means

$$
\forall \varepsilon>0 \forall \tilde{\varepsilon}>0 \exists \tau_{0}>0 \forall 0<\tau<\tau_{0}: \mathbb{P}\left[\left|X^{\tau}-X\right| \leq \varepsilon\right] \geq 1-\tilde{\varepsilon} .
$$


We state the following lemmas, which will be used in this paper.

Lemma 1. If random elements $Y^{1}, \ldots, Y^{N}$ are exchangeable, and $Z^{1}, \ldots, Z^{N}$ are also exchangeable, and independent from $Y^{1}, \ldots, Y^{N}$, then $Y^{1}+Z^{1}, \ldots, Y^{N}+Z^{N}$ are exchangeable.

Lemma 2. If random elements $Y^{1}, \ldots, Y^{N}$ are exchangeable, and

$$
Z^{k}=F\left(Y^{1}, \ldots, Y^{N}, Y^{k}\right)
$$

where $F$ is measurable and permutation invariant in the first $N$ arguments, then $Z^{1}, \ldots, Z^{N}$ are also exchangeable.

For the proof of the previous two lemmas, we refer to [26].

Lemma 3 (Uniform integrability). If $\left(X^{k}\right)$ is a bounded sequence in $L^{p}$ and $X^{k} \stackrel{\mathrm{P}}{\rightarrow} X$, then $\left\|X^{k}-X\right\|_{q} \rightarrow 0$ for all $1 \leq q<p$.

Proof. The proof is an exercise on uniform integrability [3, page 338]: Let $1 \leq q<p$. The sequence $\left(E\left(\left|X^{k}-X\right|^{q(p / q)}\right)\right)$ is bounded and $p / q>1$, thus the sequence $\left(\left|X^{k}-X\right|^{q}\right)$ is uniformly integrable. Since $\left|X^{k}-X\right| \stackrel{\mathrm{P}}{\rightarrow} 0$, and thus $\left|X^{k}-X\right|^{q} \stackrel{\mathrm{P}}{\rightarrow} 0$, it follows that $E\left(\left|X^{k}-X\right|^{q}\right) \rightarrow 0$.

Lemma 4 (Continuous mapping theorem). Let $X^{k}$ be a sequence of random elements with values on a metric space $\mathcal{A}$, such that $X^{k} \stackrel{\mathrm{P}}{\rightarrow} X$. Let $f$ be a continuous function from $\mathcal{A}$ to another metric space $\mathcal{B}$. Then $f\left(X^{k}\right) \stackrel{\mathrm{P}}{\rightarrow} f(X)$.

We refer to [33, Theorem 2.3] for a proof.

\section{The nonlinear data assimilation problem}

Consider the following classical system of stochastic equations with additive Gaussian noise, which appears in different fields, such as weather forecasting and hydrology,

$$
\begin{aligned}
X_{0} & \sim N\left(x_{\mathrm{b}}, B\right) \\
X_{i} & =\mathcal{M}_{i}\left(X_{i-1}\right)+\mu_{i}+V_{i}, \quad V_{i} \sim N\left(0, Q_{i}\right), \quad i=1, \ldots k \\
y_{i} & =\mathcal{H}_{i}\left(X_{i}\right)+W_{i}, \quad W_{i} \sim N\left(0, R_{i}\right), \quad i=1, \ldots k,
\end{aligned}
$$

with independent perturbations $V_{i}$ and $W_{i}$. The operators $\mathcal{M}_{i}$ and $\mathcal{H}_{i}$ are the model operators and the observation operators, respectively, and they are assumed to be continuously differentiable. When they are linear, we denote them by $M_{i}$ and $H_{i}$, respectively. The index $i$ denotes the time index and $k$ denotes the number of time steps. While the outputs $y_{i}$ are observed, the state $X_{i}$ and the noise variables $V_{i}$ and $W_{i}$ are hidden. The quantities $B, Q_{i}$ and $R_{i}$ are the covariance matrices of $X_{0}, V_{i}$ and $W_{i}$ respectively. The quantity $\mu_{i}$ is a deterministic vector. The objective is to estimate the hidden states $X_{1}, \ldots, X_{k}$.

Definition 3. The distribution of $X_{k}$ from (1)-(3) conditioned on $y_{1}, \ldots, y_{k-1}$ is called prior distribution. The filtering, or posterior, distribution is the distribution of $X_{k}$, conditioned on the observations of the data $y_{1}, \ldots y_{k}$. The smoothing distribution is the joint distribution of $X_{0}, \ldots, X_{k}$, conditioned on the observations of data $y_{1}, \ldots y_{k}$.

In geosciences, the prior is usually called forecast and the posterior is called analysis. In Table 1 , we collect the notation for state vectors and their ensembles for reference. 


\begin{tabular}{|c|c|c|c|}
\hline Symbol & Random & First used in & \\
\hline$X_{i}$ & yes & the state at time $i$ & 3 \\
\hline$X_{i \mid \ell}$ & no & the mean of $X_{i}$ given data $y_{1: \ell}$ & 4.1 \\
\hline$P_{i \mid \ell}$ & no & the covariance of $X_{i}$ given data $y_{1: \ell}$ & 4.1 \\
\hline$X_{i \mid \ell}^{n}$ & yes & member $n$ of an ensemble approximating $X_{i}$ given $y_{1: \ell}$ & 4.2 \\
\hline $\bar{X}_{i \mid \ell}^{N}$ & yes & the sample mean of the ensemble $X_{i \mid \ell}^{1}, \ldots, X_{i \mid \ell}^{N}$ & 4.2 \\
\hline$P_{i \mid \ell}^{N}$ & yes & the sample covariance of the ensemble $X_{i \mid \ell}^{1}, \ldots, X_{i \mid \ell}^{N}$ & 4.2 \\
\hline$U_{i \mid \ell}^{n}$ & yes & member $n$ of a reference ensemble approximating $X_{i}$ given $y_{1: \ell}$ & 4.3 \\
\hline$X_{0: i}$ & yes & composite state $\left[X_{0}, \ldots, X_{i}\right]$ at times $0, \ldots, i$ & 5.1 \\
\hline$X_{0: i \mid \ell}$ & no & the mean of $X_{0: i}$ & 5.1 \\
\hline$P_{0: i \mid \ell}$ & no & the covariance of $X_{0: i}$ & 5.1 \\
\hline$X_{0: i \mid \ell}^{n}$ & yes & member $n$ of ensemble approximating $X_{i}$ given $y_{1: \ell}$ & 5.2 \\
\hline $\bar{X}_{0: i \mid \ell}^{N}$ & yes & the sample mean of the ensemble $X_{0: i \mid \ell}^{1}, \ldots, X_{0: i \mid \ell}^{N}$ & 5.2 \\
\hline$P_{0: i, 0: i \mid \ell}^{N}$ & yes & the sample covariance of the ensemble $X_{0: i \mid \ell}^{1}, \ldots, X_{0: i \mid \ell}^{N}$ & 5.2 \\
\hline$U_{0: i \mid \ell}^{n}$ & yes & member $n$ of reference ensemble approximating $X_{i}$ given $y_{1: \ell}$ & 5.3 \\
\hline$x_{\mathrm{b}}$ & no & the background state & 6.1 \\
\hline$x_{i}$ & no & the unknown state in 4DVAR minimization & 6.1 \\
\hline$x_{0: k}$ & no & the unknown composite state in the 4 DVAR minimization & 6.1 \\
\hline$x_{i}^{j}, x_{0: k}^{j}$ & no & the iterate $j$ in the 4 DVAR minimization & 6.2 \\
\hline$X_{0: i \mid \ell}^{j, n}$ & yes & member $n$ of ensemble approximating $x_{0: i}^{j}$ & 6.3 \\
\hline $\bar{X}_{0: i \mid \ell}^{j, N}$ & yes & the sample mean of the ensemble $X_{0: i \mid \ell}^{j, 1}, \ldots, X_{0: i \mid \ell}^{j, N}$ using & 6.3 \\
\hline$X_{0: k \mid k}^{j, n, N_{j}}$ & yes & member $n$ from ensemble of size $N_{j}$ & 6.3 \\
\hline$X_{0: i \mid \ell}^{j, n, \tau}$ & yes & member $n$ of the ensemble approximating $x_{0: i}^{j}$ with step $\tau$ & 6.4 \\
\hline $\bar{X}_{0: i \mid \ell}^{j, n, \tau}$ & yes & the sample mean of the ensemble $X_{0: i \mid \ell}^{j, 1, \tau}, \ldots, X_{0: i \mid \ell}^{j, N}$ & 6.4 \\
\hline$\Delta_{i \mid \ell}^{j, n}$ & yes & 4DVAR increment ensemble members $X_{i \mid \ell}^{j, n}-x_{i}^{j-1, n}$ & 6.4 \\
\hline
\end{tabular}

Table 1: Notation for state vectors.

\section{Kalman filtering}

\subsection{Kalman filter}

The Kalman filter [13] provides an efficient computational recursive means to estimate the state of the process $X_{k}$ in the linear case, i.e., when $\mathcal{M}_{i}$ and $\mathcal{H}_{i}, i=1, \ldots, k$, are linear. Denote the mean and the covariance of $X_{i}$ given the data $y_{1}, \ldots, y_{\ell}$, by

$$
X_{i \mid \ell}=E\left(X_{i} \mid y_{1}, \ldots, y_{\ell}\right), \quad P_{i \mid \ell}=P\left(X_{i} \mid y_{1}, \ldots, y_{\ell}\right),
$$

respectively. In the linear case, the probability distribution of the process $X_{k}$ given the data up to the time $k$ is Gaussian, therefore it is characterized by its mean and covariance matrix, which can be computed as follows. 
Algorithm 1 (Kalman filter). For $i=0$, set $X_{0 \mid 0}=x_{\mathrm{b}}$ and $P_{0 \mid 0}=B$. For $i=1, \ldots, k$,

$$
\begin{aligned}
X_{i \mid i-1} & =M_{i} X_{i-1 \mid i-1}+\mu_{i}, \quad \text { (advance the mean in time) } \\
P_{i \mid i-1} & =M_{i} P_{i-1 \mid i-1} M_{i}^{\mathrm{T}}+Q_{i}, \quad \text { (advance the covariance in time) } \\
K_{i} & =P_{i \mid i-1} H_{i}^{\mathrm{T}}\left(H_{i} P_{i \mid i-1} H_{i}^{\mathrm{T}}+R_{i}\right)^{-1} \text { (the Kalman gain) } \\
X_{i \mid i} & =X_{i \mid i-1}+K_{i}\left(y_{i}-H_{i} X_{i \mid i-1}\right), \text { (update the mean from the observation } i \text { ) } \\
P_{i \mid i} & =\left(I-K_{i} H_{i}\right) P_{i \mid i-1} \quad \text { (update the covariance from the observation } i \text { ) }
\end{aligned}
$$

In atmospheric sciences, the update (5)-(6) is referred to as the analysis step.

Lemma 5. The distribution $N\left(X_{k \mid k}, P_{k \mid k}\right)$ from the Kalman filter is the filtering distribution.

See, e.g., $[1,30]$ for the proof.

If the dimension of the hidden state $X_{k}$ is large, the covariance matrices $P_{k \mid k-1}$ and $P_{k \mid k}$ are large dense matrices, hence storing such matrices in memory with the current hardware is almost impossible, and the matrix products in the computation of $P_{k \mid k-1}$ are also problematic. To solve these problems, the idea is to use ensemble methods.

\subsection{Ensemble Kalman filter (EnKF)}

The idea behind the ensemble Kalman filter is to use Monte Carlo samples and the corresponding empirical covariance matrix instead of the forecast covariance matrix $P_{k \mid k-1}$ [8]. Denote by $n$ the ensemble member index, $n=1, \ldots, N$.

Algorithm $2(\mathrm{EnKF})$. For $i=0, X_{0 \mid 0}^{n} \sim N\left(x_{\mathrm{b}}, B\right)$. For $i=1, \ldots, k$, given an analysis ensemble $X_{i-1 \mid i-1}^{1}, \ldots, X_{i-1 \mid i-1}^{N}$ at time $i-1$, the ensemble at time $i$ is built as

$$
\begin{aligned}
X_{i \mid i-1}^{n} & =M_{i} X_{i-1 \mid i-1}^{n}+\mu_{i}+V_{i}^{n}, \quad V_{i}^{n} \sim N\left(0, Q_{i}\right), \\
X_{i \mid i}^{n} & =X_{i \mid i-1}^{n}+P_{i \mid i-1}^{N} H_{i}^{\mathrm{T}}\left(H_{i} P_{i \mid i-1}^{N} H_{i}^{\mathrm{T}}+R_{i}\right)^{-1}\left(y_{i}-W_{i}^{n}-H_{i} X_{i \mid i-1}^{n}\right) \quad W_{i}^{n} \sim N\left(0, R_{i}\right),
\end{aligned}
$$

where $P_{i \mid i-1}^{N}$ is the covariance estimate from the ensemble $\left[X_{i \mid i-1}^{n}\right]_{n=1}^{N}$ ',

$$
P_{i \mid i-1}^{N}=\frac{1}{N-1} \sum_{n=1}^{N}\left(X_{i \mid i-1}^{n}-\bar{X}_{i \mid i-1}^{N}\right)\left(X_{i \mid i-1}^{n}-\bar{X}_{i \mid i-1}^{N}\right)^{\mathrm{T}} \text {, where } \bar{X}_{i \mid i-1}^{N}=\frac{1}{N} \sum_{n=1}^{N} X_{i \mid i-1}^{n} .
$$

The empirical covariance matrix $P_{i \mid i-1}^{N}$ is never computed or stored, indeed to compute the matrix products $P_{i \mid i-1}^{N} H_{i}^{\mathrm{T}}$ and $H_{i} P_{i \mid i-1}^{N} H_{i}^{\mathrm{T}}$ only matrix-vector products are needed:

$$
\begin{aligned}
P_{i \mid i-1}^{N} H_{i}^{\mathrm{T}} & =\frac{1}{N-1} \sum_{n=1}^{N}\left(X_{i \mid i-1}^{n}-\bar{X}_{i \mid i-1}^{N}\right)\left(X_{i \mid i-1}^{n}-\bar{X}_{i \mid i-1}^{N}\right)^{\mathrm{T}} H_{i}^{\mathrm{T}} \\
& =\frac{1}{N-1} \sum_{n=1}^{N}\left(X_{i \mid i-1}^{n}-\bar{X}_{i \mid i-1}^{N}\right) h_{n}^{\mathrm{T}}, \\
H_{i} P_{i \mid i-1}^{N} H_{i}^{\mathrm{T}} & =H_{i} \frac{1}{N-1} \sum_{n=1}^{N}\left(X_{i \mid i-1}^{n}-\bar{X}_{i \mid i-1}^{N}\right)\left(X_{i \mid i-1}^{n}-\bar{X}_{i \mid i-1}^{N}\right)^{\mathrm{T}} H_{i}^{\mathrm{T}}=\frac{1}{N-1} \sum_{n=1}^{N} h_{n} h_{n}^{\mathrm{T}},
\end{aligned}
$$


where

$$
h_{n}=H_{i}\left(X_{i \mid i-1}^{n}-\bar{X}_{i \mid i-1}^{N}\right)
$$

Note that the i.i.d. random vectors $\left(V_{i}^{1}, \ldots, V_{i}^{N}\right)$ are simulated here with the same statistics as the additive Gaussian noise $V_{i}$ in the original state in eq. (2). The i.i.d. random vectors $\left(W_{i}^{1}, \ldots, W_{i}^{N}\right)$ are simulated here with the same statistics as the additive Gaussian noise $W_{i}$ in the original state in eq. (3). The initial ensemble $\left[X_{0 \mid 0}\right]_{n=1}^{N}$ is simulated as i.i.d. Gaussian random vectors with mean $x_{\mathrm{b}}$ and covariance $B$, i.e. with the same statistics as the initial state $X_{0}$.

\subsection{Convergence of the EnKF}

For theoretical purposes, we define an auxiliary ensemble $U_{i \mid i}=\left[U_{i \mid i}^{n}\right]_{n=1}^{N}, i=0, \ldots, k$, called the reference ensemble, in the same way as the ensemble $X_{i \mid i}=\left[X_{i \mid i}^{n}\right]_{n=1}^{N}$, but this time for the updates of the ensemble $U_{i \mid i}$ we use the exact covariances instead of their empirical estimates. The realizations of the random perturbations $V_{i}^{n}$ and $W_{i}^{n}$ in both ensembles are the same. Thus, for $i=0, U_{0 \mid 0}^{n}=X_{0 \mid 0}^{n}$ and for $i=1, \ldots, k$, we build $U_{i \mid i}$ up to time $i$ conditioned on observations up to time $i$,

$$
\begin{aligned}
U_{i \mid i-1}^{n} & =M_{i} U_{i-1 \mid i-1}^{n}+\mu_{i}+V_{i}^{n}, \quad V_{i}^{n} \sim N\left(0, Q_{i}\right), \quad n=1, \ldots, N, \\
U_{i \mid i}^{n} & =U_{i \mid i-1}^{n}+P_{i \mid i-1} H_{i}^{\mathrm{T}}\left(H_{i} P_{i \mid i-1} H_{i}^{\mathrm{T}}+R_{i}\right)^{-1}\left(y_{i}-W_{i}^{n}-H_{i} U_{i \mid i-1}^{n}\right),
\end{aligned}
$$

where $W_{i}^{n} \sim N\left(0, R_{i}\right)$ is a random perturbation, and $P_{i \mid i-1}$ is the covariance of $U_{i \mid i-1}^{1}$

$$
P_{i \mid i-1}=E\left[\left(U_{i \mid i-1}^{n}-E\left(U_{i \mid i-1}^{n}\right)\right)\left(U_{i \mid i-1}^{n}-E\left(U_{i \mid i-1}^{n}\right)\right)^{\mathrm{T}}\right] .
$$

Note that the only difference between the two ensembles $X_{k \mid k}$ and $U_{k \mid k}$ is that for the construction of $X_{k \mid k}$, we use the empirical prediction covariance $P_{k \mid k-1}^{N}$ of the ensemble, which depends on all ensemble members, instead of the exact covariance. Therefore, $X_{k \mid k}^{n}, n=1, \ldots, N$, are in general dependent. On the other hand:

Lemma 6. The members of the ensemble $\left[U_{k \mid k}^{n}\right]_{n=1}^{N}$ are i.i.d and the distribution of each $U_{k \mid k}^{n}$ is the same as the filtering distribution.

Proof. The proof is by induction and the same as in [26, Lemma 4], except we take the additional perturbation $V_{k}^{n}$ into account. Since $\left[V_{k}^{n}\right]_{n=1}^{N}$ are Gaussian and independent of everything else by assumption, $\left[U_{k \mid k}^{n}\right]_{n=1}^{N}$ are independent and Gaussian. The forecast covariance $P_{k \mid k-1}$ is constant (non-random), and, consequently, the analysis step (13) is a linear transformation, which preserves the independence of the ensemble members and the Gaussianity of the distribution. It is known that the members of the reference ensemble have the same mean and covariance as given by the Kalman filter [6, eq. (15) and (16)]. The proof is completed by noting that a Gaussian distribution is determined by its mean and covariance.

Theorem 3. For any $i=0, \ldots, k$, the random matrix

$$
\left[\begin{array}{c}
X_{i \mid i}^{1}, \ldots, X_{i \mid i}^{N} \\
U_{i \mid i}^{1}, \ldots, U_{i \mid i}^{N}
\end{array}\right]
$$


has exchangeable columns, and

$$
X_{i \mid i}^{1} \rightarrow U_{i \mid i}^{1}
$$

in all $L^{p}, 1 \leq p<\infty$, as $N \rightarrow \infty$. Also,

$$
\begin{aligned}
\bar{X}_{i \mid i-1}^{N} & =\frac{1}{N} \sum_{n=1}^{N} X_{i \mid i}^{n} \rightarrow E\left(U_{i \mid i}^{1}\right), \\
P_{i \mid i-1}^{N} & =\frac{1}{N-1} \sum_{n=1}^{N}\left(X_{i \mid i-1}^{n}-\bar{X}_{i \mid i-1}^{N}\right)\left(X_{i \mid i-1}^{n}-\bar{X}_{i \mid i-1}^{N}\right)^{\mathrm{T}} \\
& \rightarrow P_{i \mid i-1}=E\left[\left(U_{i \mid i-1}^{1}-E\left(U_{i \mid i-1}^{1}\right)\right)\left(U_{i \mid i-1}^{1}-E\left(U_{i \mid i-1}^{1}\right)\right)^{\mathrm{T}}\right],
\end{aligned}
$$

in all $L^{p}, 1 \leq p<\infty$, as $N \rightarrow \infty$.

Proof. The theorem is again a simple extension of that of [26, Theorem 1], by adding the model error $V_{i}^{n}$ in each step of the induction over $i$.

Note that since (14) has exchangeable columns and $X_{i \mid i}^{1} \rightarrow U_{i \mid i}^{1}$ in $L^{p}$, we have the same convergence result for every fixed $n, X_{i \mid i}^{n} \rightarrow U_{i \mid i}^{n}$ in all $L^{p}$, as $N \rightarrow \infty$.

\section{$5 \quad$ Kalman smoothing}

\subsection{Kalman smoother (KS)}

A smoother estimates the composite hidden state

$$
X_{0: i}=\left[\begin{array}{c}
X_{0} \\
\vdots \\
X_{i}
\end{array}\right]
$$

given all observations $y_{1}, \ldots, y_{i}$. Again, the Kalman smoother provides the exact result in the linear Gaussian case. Denote by $X_{0: i \mid \ell}$ the expectation of the composite state $X_{0: i}$ given the observations $y_{1}, \ldots, y_{\ell}$, and by $P_{0: i \mid \ell}$ the corresponding covariance. In the linear case, we write the stochastic system (2)-(3) in terms of the composite state $X_{0: i}$ as

$$
\begin{aligned}
& X_{0: i}=\left[\begin{array}{cccc}
I_{m} & 0 & \ldots & 0 \\
0 & I_{m} & \vdots & \vdots \\
\vdots & \ddots & \ddots & 0 \\
0 & \ldots & \ddots & I_{m} \\
0 & \ldots & 0 & M_{i}
\end{array}\right] X_{0: i-1}+\left[\begin{array}{c}
0 \\
\vdots \\
\mu_{i}
\end{array}\right]+\left[\begin{array}{c}
0 \\
\vdots \\
V_{i}
\end{array}\right] \\
& =\left[\begin{array}{c}
I_{m(i-1)}^{(i)} \\
\tilde{M}_{i}
\end{array}\right] X_{0: i-1}+\left[\begin{array}{c}
0 \\
\vdots \\
\mu_{i}
\end{array}\right]+\left[\begin{array}{c}
0 \\
\vdots \\
V_{i}
\end{array}\right], \quad V_{i} \sim N\left(0, Q_{i}\right), \\
& y_{i}=\left[0, \ldots, H_{i}\right] X_{0: i}+W_{i}=\tilde{H}_{i} X_{0: i}+W_{i}, \quad W_{i} \sim N\left(0, R_{i}\right) \text {, }
\end{aligned}
$$


where $m$ is the dimension of the state $X_{i}, I_{d}$ is the identity matrix in $\mathbf{R}^{d \times d}$, and

$$
\tilde{H}_{i}=\left[0, \ldots, H_{i}\right], \quad \tilde{M}_{i}=\left[0, \ldots, M_{i}\right] .
$$

Applying the Kalman filter analysis step (4)-(6) to the observation (16) of the composite state $X_{0: i}$, we obtain the Kalman smoother:

$$
\begin{aligned}
& X_{0: i \mid i-1}=\left[\begin{array}{c}
I_{m(i-1)} \\
\tilde{M}_{i}
\end{array}\right] X_{0: i-1 \mid i-1}+\left[\begin{array}{c}
0 \\
\vdots \\
\mu_{i}
\end{array}\right]=\left[\begin{array}{c}
X_{0: i-1 \mid i-1} \\
M_{i} X_{i-1, i-1}+\mu_{i}
\end{array}\right] \text {, } \\
& P_{0: i \mid i-1}=\left[\begin{array}{c}
I_{m(i-1)} \\
\tilde{M}_{i}
\end{array}\right] P_{0: i-1 \mid i-1}\left[\begin{array}{c}
I_{m(i-1)} \\
\tilde{M}_{i}
\end{array}\right]^{\mathrm{T}}+\left[\begin{array}{cc}
0 & 0 \\
0 & Q_{i}
\end{array}\right] \\
& =\left[\begin{array}{cc}
P_{0: i-1 \mid i-1} & P_{0: i-1 \mid i-1} \tilde{M}_{i}^{\mathrm{T}} \\
\tilde{M}_{i} P_{0: i-1 \mid i-1} & \tilde{M}_{i} P_{0: i-1 \mid i-1} \tilde{M}_{i}^{\mathrm{T}}+Q_{i}
\end{array}\right] \text {, } \\
& K_{i}=P_{0: i \mid i-1} \tilde{H}_{i}^{\mathrm{T}}\left(R_{i}+\tilde{H}_{i} P_{0: i \mid i-1} \tilde{H}_{i}^{\mathrm{T}}\right)^{-1} \\
& =P_{0: i \mid i-1} \tilde{H}_{i}^{\mathrm{T}}\left(R_{i}+H_{i} P_{i, i \mid i-1} H_{i}^{\mathrm{T}}\right)^{-1}, \\
& X_{0: i \mid i}=X_{0: i \mid i-1}+K_{i}\left(y_{i}-\tilde{H}_{i} X_{0: i \mid i-1}\right)=X_{0: i \mid i-1}+K_{i}\left(y_{i}-H_{i} X_{i \mid i-1}\right), \\
& P_{0: i \mid i}=\left(I_{m i}-K_{i} \tilde{H}_{i}\right) P_{0: i \mid i-1} \text {. }
\end{aligned}
$$

Lemma 7. The distribution $N\left(X_{0: k \mid k}, P_{0: k, 0: k \mid k}\right)$ from the Kalman smoother is the smoothing distribution, and its mean $X_{0: k \mid k}$ is the solution of the least squares problem,

$$
X_{0: k \mid k}=\underset{x_{0: k}}{\operatorname{argmin}}\left(\left|x_{0}-x_{\mathrm{b}}\right|_{B^{-1}}^{2}+\sum_{i=1}^{k}\left|x_{i}-M_{i} x_{i-1}-\mu_{i}\right|_{Q_{i}^{-1}}^{2}+\sum_{i=1}^{k}\left|y_{i}-H_{i} x_{i}\right|_{R_{i}^{-1}}^{2}\right) .
$$

Proof. The mean $X_{0: k \mid k}$ maximizes the joint posterior probability density of the composite state $X_{0: k}$ given $y_{1: k}$, which is proportional to

$$
e^{-\frac{1}{2}\left|x_{0}-x_{\mathrm{b}}\right|_{B}^{2}} e^{-\frac{1}{2} \sum_{i=1}^{k}\left|x_{i}-M_{i} x_{i-1}-\mu_{i}\right|_{Q_{i}^{-1}}^{2}} e^{-\frac{1}{2} \sum_{i=1}^{k}\left|y_{i}-H_{i} x_{i}\right|_{R_{i}^{-1}}^{2}}
$$

from the Bayes theorem.

Again, when $m$ is large, the covariance matrices $P_{0: i \mid i-1}$ and $P_{0: i \mid i}$ are very large and the matrix products in the computation of $P_{0: i \mid i-1}$ is also problematic to implement, and we turn to ensemble methods.

\subsection{Ensemble Kalman smoother (EnKS)}

In the ensemble Kalman smoother [8], the covariances are replaced by approximations from the ensemble. Let

$$
\left[\left[\begin{array}{c}
X_{0 \mid j}^{1} \\
\vdots \\
X_{i \mid j}^{1}
\end{array}\right], \ldots,\left[\begin{array}{c}
X_{0 \mid j}^{N} \\
\vdots \\
X_{i \mid j}^{N}
\end{array}\right]\right]=\left[X_{0: i \mid j}^{1}, \ldots, X_{0: i \mid j}^{N}\right]=\left[X_{0: i \mid j}^{n}\right]_{n=1}^{N}
$$


denote an ensemble of $N$ model states over time up to $i$, conditioned on the observations up to time $j$.

Algorithm 4 (EnKS). For $i=0$, the ensemble $\left[X_{0 \mid 0}^{n}\right]_{n=1}^{N}$ consists of i.i.d. Gaussian random variables

$$
X_{0 \mid 0}^{n} \sim N\left(x_{\mathrm{b}}, B\right) .
$$

For $i=1, \ldots, k$, advance the model to time $i$ by

$$
X_{i \mid i-1}^{n}=M_{i} X_{i-1 \mid i-1}^{n}+\mu_{i}+V_{i}^{n}, \quad V_{i}^{n} \sim N\left(0, Q_{i}\right), \quad n=1, \ldots, N .
$$

Incorporate the observation at time $i$,

$$
y_{i}=\tilde{H}_{i} X_{i}+W_{i}, \quad W_{i} \sim N\left(0, R_{i}\right)
$$

into the ensemble of composite states $\left[X_{0: i \mid i-1}^{1}, \ldots, X_{0: i \mid i-1}^{N}\right]$ in the same way as for the EnKF update,

$$
X_{0: i \mid i}^{n}=X_{0: i \mid i-1}^{n}+P_{0: i \mid i-1}^{N} \tilde{H}_{i}^{\mathrm{T}}\left(\tilde{H}_{i} P_{0: i \mid i-1}^{N} \tilde{H}_{i}^{\mathrm{T}}+R_{i}\right)^{-1}\left(y_{i}-W_{i}^{n}-H_{i} X_{i \mid i-1}^{n}\right)
$$

where $P_{0: i \mid i-1}^{N}$ is a covariance estimate from the ensemble $X_{0: i \mid i-1}$ and $W_{i}^{n} \sim N\left(0, R_{i}\right)$ are random perturbations. Similarly as in (9)-(11), only the following matrix-vector products are needed:

$$
\begin{aligned}
P_{0: i \mid i-1}^{N} \tilde{H}_{i}^{\mathrm{T}} & =\frac{1}{N-1} \sum_{n=1}^{N}\left(X_{0: i \mid i-1}^{n}-\bar{X}_{0: i \mid i-1}^{N}\right)\left(X_{i \mid i-1}^{n}-\bar{X}_{i \mid i-1}^{N}\right)^{\mathrm{T}} H_{i}^{\mathrm{T}} \\
& =\frac{1}{N-1} \sum_{n=1}^{N}\left(X_{0: i \mid i-1}^{n}-\bar{X}_{0: i \mid i-1}^{N}\right) h_{n}^{\mathrm{T}}, \\
\tilde{H}_{i}^{\mathrm{T}} P_{0: i \mid i-1} \tilde{H}_{i}^{\mathrm{T}} & =H_{i} \frac{1}{N-1} \sum_{n=1}^{N}\left(X_{i \mid i-1}^{n}-\bar{X}_{i \mid i-1}^{N}\right)\left(X_{i \mid i-1}^{n}-\bar{X}_{i \mid i-1}^{N}\right)^{\mathrm{T}} H_{i}^{\mathrm{T}}=\frac{1}{N-1} \sum_{n=1}^{N} h_{n} h_{n}^{\mathrm{T}},
\end{aligned}
$$

where again

$$
h_{n}=H_{i}\left(X_{i \mid i-1}^{n}-\bar{X}_{i \mid i-1}^{N}\right)
$$

and

$$
\bar{X}_{\ell \mid i-1}^{N}=\frac{1}{N} \sum_{n=1}^{N} X_{\ell \mid i-1}^{n} .
$$

\subsection{Convergence of the EnKS}

Just as for the EnKF, we construct an ensemble $U_{0: k \mid k}=\left[U_{0: k \mid k}^{n}\right]_{n=1}^{N}$ in the same way as the ensemble $\left[X_{0: k \mid k}^{n}\right]_{n=1}^{N}$, but for the updates of the ensemble $U_{0: k \mid k}$ we use the exact covariances instead of their empirical estimates. So, for $i=0, U_{0 \mid 0}^{n}=X_{0 \mid 0}^{n}$, and for $i=1, \ldots, k, n=1, \ldots, N$,

$$
\begin{aligned}
& U_{i \mid i-1}^{n}=M_{i} U_{i-1 \mid i-1}^{n}+\mu_{i}+V_{i}^{n}, \quad V_{i}^{n} \sim N\left(0, Q_{i}\right), \\
& U_{0: i \mid i}^{n}=U_{0: i \mid i-1}^{n}+P_{0: i \mid i-1} \tilde{H}_{i}^{\mathrm{T}}\left(\tilde{H}_{i} P_{0: i \mid i-1} \tilde{H}_{i}^{\mathrm{T}}+R_{i}\right)^{-1}\left(y_{i}-W_{i}^{n}-H_{i} U_{i \mid i-1}^{n}\right), \\
& \quad W_{i}^{n} \sim N\left(0, R_{i}\right),
\end{aligned}
$$


where $P_{0: i \mid i-1}$ is the covariance of $U_{0: i \mid i-1}^{1}$.

Since the Kalman smoother is nothing else than the Kalman filter for the composite state $X_{0: k}$, the same induction step as in Theorem 3 applies for each $i$, and we have the following.

Lemma 8. The random elements $U_{0: k \mid k}^{1}, \ldots, U_{0: k \mid k}^{N}$ are i.i.d and the distribution of each $U_{0: k \mid k}^{n}$ is the same as the smoothing distribution. In particular, $E\left(U_{0: k \mid k}\right)=X_{0: k \mid k}$, with $X_{0: k \mid k}$ is the least squares solution (18).

Theorem 5. For each time step $i=0, \ldots, k$, the random matrix

$$
\left[\begin{array}{c}
X_{0: i \mid i}^{1}, \ldots, X_{0: i \mid i}^{N} \\
U_{0: i \mid i}^{1}, \ldots, U_{0: i \mid i}^{N}
\end{array}\right] \text {. }
$$

has exchangeable columns, and $X_{0: i \mid i}^{1} \rightarrow U_{0: i \mid i}^{1}, \bar{X}_{0: i \mid i}^{N} \rightarrow E\left(U_{0: i \mid i}^{1}\right)$, and $P_{0: i \mid i}^{N} \rightarrow P_{0: i \mid i}$ in $L^{p}$ as $N \rightarrow$ $\infty$, for all $1 \leq p<\infty$

\section{$6 \quad$ Variational data assimilation and 4DVAR}

\subsection{DVAR as an optimization problem}

We estimate the compound state $X_{0: k}$ of the stochastic system (1)-(3), conditioned on the observations $y_{1}, \ldots, y_{k}$, by the maximum posterior probability density,

$$
\mathbb{P}\left(x_{0: k} \mid y_{1: k}\right) \propto e^{-\frac{1}{2}\left(\left|x_{0}-x_{\mathrm{b}}\right|_{B^{-1}}^{2}+\sum_{i=1}^{k}\left|x_{i}-\mathcal{M}_{i}\left(x_{i-1}\right)-\mu_{i}\right|_{Q_{i}^{-1}}^{2}+\sum_{i=1}^{k}\left|y_{i}-\mathcal{H}_{i}\left(x_{i}\right)\right|_{R_{i}^{-1}}^{2}\right)} \rightarrow \max _{x_{0: k}},
$$

which is the same as solving the nonlinear least squares problem for the composite state $x_{0: k}$,

$$
\left|x_{0}-x_{\mathrm{b}}\right|_{B^{-1}}^{2}+\sum_{i=1}^{k}\left|x_{i}-\mathcal{M}_{i}\left(x_{i-1}\right)-\mu_{i}\right|_{Q_{i}^{-1}}^{2}+\sum_{i=1}^{k}\left|y_{i}-\mathcal{H}_{i}\left(x_{i}\right)\right|_{R_{i}^{-1}}^{2} \rightarrow \min _{x_{0: k}} .
$$

Numerical solution of the nonlinear least squares problem (26) is the essence of weak-constraint 4-dimensional variational data assimilation (4DVAR) $[9,31]$.

\subsection{The Levenberg-Marquardt method and incremental 4DVAR}

Consider an approximate solution $x_{0: k}^{j-1}$ of the nonlinear least squares problem (26). We seek a better approximation $x_{0: k}^{j}$. Linearizing the model and the observation operators at $x_{0: k}^{j-1}$ by their tangent operators and adding a penalty term to control the size of the increment $x_{0: k}^{j}-x_{0: k}^{j-1}$, yields the linear least squares problem for $x_{0: k}^{j}$ in the Levenberg-Marquardt (LM) method [20, 27] for the solution of the nonlinear least squares (26).

Algorithm 6 (LM method). Given $x_{0: k}^{0}$ and $\gamma \geq 0$, compute the iterations $x_{0: k}^{j}$ for $j=1,2, \ldots$, as the solutions of the least squares problem linearized at $x_{0: k}^{j-1}$,

$$
\begin{aligned}
x_{0: k}^{j} & =\underset{x_{0: k}}{\operatorname{argmin}}\left|x_{0}-x_{b}\right|_{B^{-1}}^{2}+\sum_{i=1}^{k}\left|x_{i}-\mathcal{M}_{i}\left(x_{i-1}^{j-1}\right)-\mathcal{M}_{i}^{\prime}\left(x_{i-1}^{j-1}\right)\left(x_{i-1}-x_{i-1}^{j-1}\right)-\mu_{i}\right|_{Q_{i}^{-1}}^{2} \\
& +\sum_{i=1}^{k}\left|y_{i}-\mathcal{H}_{i}\left(x_{i}^{j-1}\right)-\mathcal{H}_{i}^{\prime}\left(x_{i}^{j-1}\right)\left(x_{i}-x_{i}^{j-1}\right)\right|_{R_{i}^{-1}}^{2}+\sum_{i=1}^{k} \gamma\left|x_{i}-x_{i}^{j-1}\right|^{2} .
\end{aligned}
$$


For $\gamma=0,(27)$ becomes the Gauss-Newton method, which can converge at a rate close to quadratic, but convergence is not guaranteed even locally. Under suitable technical assumptions, the LM method is guaranteed to converge globally if the regularization parameter $\gamma$ is large enough $[28,10]$, and a suitable sequence of penalty parameters $\gamma_{j} \geq 0$ changing from step to step can be found adaptively. The LM method is a precursor of the trust-region method in the sense that it seeks to determine when the faster Gauss-Newton method $(\gamma=0)$ is applicable and when it is not and should be blended with a slower but safer gradient descent method $(\gamma>0)$. In this paper, we consider only the case of a constant penalty parameter $\gamma>0$.

The Gauss-Newton method for the solution of nonlinear least squares is known in atmospheric sciences as incremental 4DVAR [7]. The use of Levenberg-Marquardt iterations was proposed by $[32]$.

\subsection{LM-EnKS with tangent operators}

From (18), it follows that the linear least squares problem (27) can be interpreted as finding the maximum posterior probability density for a linear stochastic system with all Gaussian probability distributions. The penalty terms $\gamma\left|x_{i}-x_{i}^{j-1}\right|^{2}$ are implemented as additional independent observations [12] of the form

$$
x_{i}^{j-1}=x_{i}+E_{i}, \quad E_{i} \sim N\left(0, \frac{1}{\gamma} I_{m}\right), \quad i=1, \ldots, k .
$$

Lemma 9. The LM iterate $x_{0: k}^{j}$, defined by (27), equals to the mean

$$
x_{0: k}^{j}=E\left(X_{0: k \mid k}^{j}\right),
$$

of the smoothing distribution of the stochastic system

$$
\begin{aligned}
X_{0}^{j} & \sim N\left(x_{\mathrm{b}}, B\right), \\
X_{i}^{j} & =\mathcal{M}_{i}^{\prime}\left(x_{i-1}^{j-1}\right)\left(X_{i-1}^{j}-x_{i-1}^{j-1}\right)+\mathcal{M}_{i}\left(x_{i-1}^{j-1}\right)+\mu_{i}+V_{i}^{j}, \quad V_{i}^{j} \sim N\left(0, Q_{i}\right) \quad i=1, \ldots, k, \\
\tilde{y}_{i} & =\tilde{\mathcal{H}}_{i}\left(x_{i}^{j-1}\right)+\tilde{\mathcal{H}}_{i}^{\prime}\left(x_{i}^{j-1}\right)\left(X_{i}^{j}-x_{i}^{j-1}\right)+\tilde{W}_{i}^{j}, \quad \tilde{W}_{i}^{j} \sim N\left(0, \tilde{R}_{i}\right), \quad i=1, \ldots, k,
\end{aligned}
$$

where

$$
\tilde{y}_{i}=\left[\begin{array}{c}
y_{i} \\
x_{i}^{j-1}
\end{array}\right], \quad \tilde{\mathcal{H}}_{i}=\left[\begin{array}{c}
\mathcal{H}_{i} \\
I_{m}
\end{array}\right], \quad \tilde{R}_{i}=\left[\begin{array}{cc}
R_{i} & 0 \\
0 & \frac{1}{\gamma} I_{m}
\end{array}\right],
$$

or, equivalently

$$
\begin{aligned}
X_{0}^{j} & \sim N\left(x_{\mathrm{b}}, B\right), \\
X_{i}^{j} & =M_{i}^{j} X_{i-1}^{j}+\tilde{\mu}_{i}^{j}+V_{i}^{j}, \quad V_{i}^{j} \sim N\left(0, Q_{i}\right) \quad i=1, \ldots, k, \\
\tilde{y}_{i}^{j} & =\tilde{H}_{i}^{j} X_{i}^{j}+\tilde{W}_{i}^{j}, \quad \tilde{W}_{i}^{j} \sim N\left(0, \tilde{R}_{i}\right), \quad i=1, \ldots, k,
\end{aligned}
$$

where

$$
\begin{aligned}
& M_{i}^{j}=\mathcal{M}_{i}^{\prime}\left(x_{i-1}^{j-1}\right), \quad \tilde{\mu}_{i}^{j}=\mathcal{M}_{i}\left(x_{i-1}^{j-1}\right)+\mu_{i}-\mathcal{M}_{i}^{\prime}\left(x_{i-1}^{j-1}\right) x_{i-1}^{j-1}, \\
& \tilde{H}_{i}^{j}=\tilde{\mathcal{H}}_{i}^{\prime}\left(x_{i}^{j-1}\right), \quad \tilde{y}_{i}^{j}=\tilde{y}_{i}+\tilde{\mathcal{H}}_{i}^{\prime}\left(x_{i}^{j-1}\right) x_{i}^{j-1}-\tilde{\mathcal{H}}_{i}\left(x_{i}^{j-1}\right) .
\end{aligned}
$$


Proof. The system (28)-(30) has the same form as the original problem (1)-(3) and all distributions are Gaussian, hence Lemma 7 applies.

Corollary 7. The LM iterate $x^{j}$ is the mean found from the Kalman smoother (15)-(17), applied to the linear stochastic system (28)-(30).

However, since the dimension of the state is generally large, we apply the EnKS (19)-(25) to solve (28)-(30) approximately. In each LM iteration $j=1,2, \ldots$, the linearized least squares solution $x^{j}$ is approximated by the sample mean $\bar{X}_{0: k \mid k}^{j, N_{j}}$ from the EnKS and the least squares are linearized at the previous iterate $\bar{X}_{0: k \mid k}^{j-1, N_{j-1}}$ rather than at $x^{j-1}$. However, for $j=0$ this notation is formal for the sake of consistency only. There is no ensemble for $j=0$.

Algorithm 8. Given an initial approximation $x_{0: k}^{0}$, and $\gamma \geq 0$. Initialize

$$
\tilde{x}^{j}=\bar{X}_{0: k \mid k}^{j, N_{j}}=x_{0: k}^{0} \quad \text { for } j=0 .
$$

LM loop: For $j=1,2, \ldots$ Choose an ensemble size $N_{j}$.

EnKS loop: For $i=0$, the ensemble $\left[X_{0 \mid 0}^{j, n}\right]_{n=1}^{N_{j}}$ consists of i.i.d. Gaussian random variables

$$
X_{0 \mid 0}^{j, n} \sim N\left(\tilde{x}_{0}^{0}, B\right) .
$$

For $i=1, \ldots, k$, advance the model in time (the forecast step) by

$$
\begin{aligned}
X_{i \mid i-1}^{j, n} & =\mathcal{M}_{i}^{\prime}\left(\bar{X}_{i-1 \mid k}^{j-1, N_{j-1}}\right)\left(X_{i-1 \mid i-1}^{j, n}-\bar{X}_{i-1 \mid k}^{j-1, N_{j-1}}\right)+\mathcal{M}_{i}\left(\bar{X}_{i-1 \mid k}^{j-1, N_{j-1}}\right)+\mu_{i}+V_{i}^{j, n}, \\
V_{i}^{j, n} & \sim N\left(0, Q_{i}\right), \quad n=1, \ldots, N_{j} .
\end{aligned}
$$

Incorporate the observations at time $i$ into the ensemble of composite states $\left[X_{0: i \mid i-1}^{j, n}\right]_{n=1}^{N_{j}}$ in the same way as in the EnKF analysis step,

$$
\begin{aligned}
& X_{0: i \mid i}^{j, n}= X_{0: i \mid i-1}^{j, n}+P_{0: i \mid i-1}^{j, N_{j}} \tilde{H}_{i}^{j \mathrm{~T}}\left(\tilde{H}_{i}^{j} P_{0: i \mid i-1}^{j, N_{j}} \tilde{H}_{i}^{j \mathrm{~T}}+\tilde{R}_{i}\right)^{-1} \\
& \cdot\left(\tilde{y}_{i}-\tilde{W}_{i}^{j, n}-\tilde{\mathcal{H}}_{i}\left(\bar{X}_{i \mid k}^{j-1, N_{j-1}}\right)-\tilde{\mathcal{H}}_{i}^{\prime}\left(\bar{X}_{i \mid k}^{j-1, N_{j-1}}\right)\left(X_{i \mid i-1}^{j, n}-\bar{X}_{i \mid k}^{j-1, N_{j-1}}\right)\right), \\
& \tilde{W}_{i}^{j, n} \sim N\left(0, \tilde{R}_{i}\right)
\end{aligned}
$$

where $P_{0: i \mid i-1}^{j, N_{j}}$ is the sample covariance from the ensemble $\left[X_{0: i \mid i-1}^{j, n}\right]_{n=1}^{N_{j}}$. Similarly as in (9)-(11), 
only the following matrix-vector products are needed:

$$
\begin{aligned}
P_{0: i \mid i-1}^{j, N_{j}} \tilde{H}_{i}^{j \mathrm{~T}} & =\frac{1}{N_{j}-1} \sum_{n=1}^{N_{j}}\left(X_{0: i \mid i-1}^{j, n}-\bar{X}_{0: i \mid i-1}^{j, N_{j}}\right)\left(X_{i \mid i-1}^{j, n}-\bar{X}_{i \mid i-1}^{j, N_{j}}\right)^{\mathrm{T}} \tilde{H}_{i}^{j \mathrm{~T}} \\
& =\frac{1}{N_{j}-1} \sum_{n=1}^{N_{j}}\left(X_{0: i \mid i-1}^{j, n}-\bar{X}_{0: i \mid i-1}^{j, N_{j}}\right) h_{i}^{j, n \mathrm{~T}}, \\
\tilde{H}_{i}^{j} P_{0: i \mid i-1}^{j, N_{j}} \tilde{H}_{i}^{j \mathrm{~T}} & =\frac{1}{N_{j}-1} \sum_{n=1}^{N_{j}} \tilde{H}_{i}^{j}\left(X_{i \mid i-1}^{j, n}-\bar{X}_{i \mid i-1}^{j, N_{j}}\right)\left(X_{i \mid i-1}^{j, n}-\bar{X}_{i \mid i-1}^{j, N_{j}}\right)^{\mathrm{T}} \tilde{H}_{i}^{j \mathrm{~T}} \\
& =\frac{1}{N_{j}-1} \sum_{n=1}^{N_{j}} h_{i}^{j, n} h_{i}^{j, n \mathrm{~T}},
\end{aligned}
$$

where

$$
h_{i}^{j, n}=\tilde{H}_{i}^{j}\left(X_{i \mid i-1}^{j, n}-\bar{X}_{i \mid i-1}^{j, N_{j}}\right)=\tilde{\mathcal{H}}_{i}^{\prime}\left(\bar{X}_{i \mid k}^{j-1, N_{j-1}}\right)\left(X_{i \mid i-1}^{j, n}-\bar{X}_{i \mid i-1}^{j, N_{j}}\right)
$$

and

$$
\bar{X}_{i \mid i-1}^{j, N_{j}}=\frac{1}{N_{j}} \sum_{n=1}^{N_{j}} X_{i \mid i-1}^{j, n}, \quad \bar{X}_{0: i \mid i-1}^{j, N_{j}}=\frac{1}{N_{j}} \sum_{n=1}^{N_{j}} X_{0: i \mid i-1}^{j, n} .
$$

The next iterate is $\tilde{x}^{j}=\bar{X}_{0: k \mid k}^{j, N_{j}}$.

In the rest of this section, we study the asymptotic behavior of Algorithm 8 when the ensembles sizes $N_{1}, \ldots N_{j} \rightarrow \infty$. We start with an a-priori $L^{p}$ bound on the ensemble members, independent of the ensemble size.

Assumption 9. The model and observation operators, $\mathcal{M}_{i}$, and $\mathcal{H}_{i}$ are continuously differentiable, with at most polynomial growth at infinity, and their Jacobians have at most polynomial growth at infinity, i.e. there exists $\kappa>0$ and $s \geq 0$, such that $\left|\mathcal{M}_{i}(x)\right| \leq \kappa\left(1+|x|^{s}\right),\left|\mathcal{M}_{i}^{\prime}(x)\right| \leq \kappa\left(1+|x|^{s}\right)$, $\left|\mathcal{H}_{i}(x)\right| \leq \kappa\left(1+|x|^{s}\right)$, and $\left|\mathcal{H}_{i}^{\prime}(x)\right| \leq \kappa\left(1+|x|^{s}\right)$ for all $i$ and all $x$.

Since we are interested in the convergence with the ensemble size, we need a notation to distinguish between $X_{0: k \mid k}^{j, n}$ coming from ensembles of different sizes $N_{j}$. Thus, when we need to make such distinction, we denote by $X_{0: k \mid k}^{j, n, N_{j}}$ the $n$-th ensemble member from the ensemble $\left[X_{0: k \mid k}^{j, 1}, \ldots, X_{0: k \mid k}^{j, N_{j}}\right]$ of size $N_{j}$ in Algorithm 8, and similarly for other subscripts and superscripts.

Lemma 10. For any $1 \leq p<\infty$, any $i=0, \ldots, k$ and any $j=1,2, \ldots$, there exists a constant $C(i, j, p)$ such that in Algorithm 8,

$$
\left\|X_{0: i \mid i}^{j, n, N_{j}}\right\|_{p} \leq C(i, j, p)
$$

for all $n=1, \ldots, N_{j}$ and all $N_{j}$.

Proof. Let $p \in[1, \infty)$. We will prove (38) by induction on the iteration number $j$. For $j=1$, $\tilde{x}^{j-1}$ is constant, otherwise, for $j \geq 2,\left\|\tilde{x}^{j-1}\right\|_{p}$ is bounded independently of the ensemble sizes by induction assumption because

$$
\tilde{x}^{j-1}=\bar{X}_{0: k \mid k}^{j-1, N_{j-1}}=\frac{1}{N_{j-1}} \sum_{n=1}^{N_{j-1}} X_{0: k \mid k}^{j-1, n, N_{j-1}} .
$$


For a fixed $j$, we now proceed by induction on the time step $i$. For $i=0, X_{0 \mid 0}^{j, n} \sim N\left(x_{0}^{0}, B\right)$, thus $\left\|X_{0 \mid 0}^{j, n}\right\|_{p}$ does not depend on $n$ or $N_{j}$. For $i=1, \ldots, k$, from (35), we have

$$
\left\|X_{i \mid i-1}^{j, n}\right\|_{p} \leq\left\|\mathcal{M}_{i}^{\prime}\left(\tilde{x}_{i-1}^{j-1}\right)\right\|_{2 p}\left(\left\|X_{i-1 \mid i-1}^{j, n}\right\|_{2 p}+\left\|\tilde{x}_{i-1}^{j-1}\right\|_{2 p}\right)+\left\|\mathcal{M}_{i}\left(\tilde{x}_{i-1}^{j-1}\right)\right\|_{p}+\left|\mu_{i}\right|+\left\|V_{i}^{j, n}\right\|_{p} .
$$

From Assumption 9 and the fact that $V_{i}^{j, n}$ is normally distributed, there exist a constant $C_{p}$ such that

$$
\begin{aligned}
\left\|X_{i \mid i-1}^{j, n}\right\|_{p} & \leq \kappa C_{p}\left(1+\left\|\tilde{x}_{i-1}^{j-1}\right\|_{2 p s}^{s}\right)\left(\left\|X_{i-1 \mid i-1}^{j, n}\right\|_{2 p}+\left\|\tilde{x}_{i-1}^{j-1}\right\|_{2 p}\right) \\
& +\kappa C_{p}\left(1+\left\|\tilde{x}_{i-1}^{j-1}\right\|_{p s}^{s}\right)+\left|\mu_{i}\right|+C_{p} .
\end{aligned}
$$

Bounding $\tilde{x}_{i-1}^{j-1}$ by the induction assumption on $j$ and $X_{i-1 \mid i-1}^{j, n}$ by the induction assumption on $i$, we have that $\left\|X_{0: i \mid i-1}^{j, n, N_{j}}\right\|_{p}$ is bounded independently of $n$ and $N_{j}$. From equation (36), and the fact that $\tilde{H}_{i}^{j}=\tilde{\mathcal{H}}_{i}^{\prime}\left(\tilde{x}_{i}^{j-1}\right)=\left[0, \ldots, \mathcal{H}_{i}^{\prime}\left(\tilde{x}_{i}^{j-1}\right)\right]$ we conclude that

$$
\begin{aligned}
\left\|X_{0: i \mid i}^{j, n}\right\|_{p} \leq & \left\|X_{0: i \mid i-1}^{j, n}\right\|_{p}+\left\|P_{0: i \mid i-1}^{j, N_{j}} \tilde{\mathcal{H}}_{i}^{\prime \mathrm{T}}\left(\tilde{x}_{i}^{j-1}\right)\left(\tilde{\mathcal{H}}_{i}^{\prime}\left(\tilde{x}_{i}^{j-1}\right) P_{0: i \mid i-1}^{j, N_{j}} \tilde{\mathcal{H}}_{i}^{\prime \mathrm{T}}\left(\tilde{x}_{i}^{j-1}\right)+\tilde{R}_{i}\right)^{-1}\right\|_{2 p} \\
& \cdot\left\|\tilde{y}_{i}-\tilde{W}_{i}^{j, n}-\mathcal{H}_{i}\left(\tilde{x}_{i}^{j-1}\right)-\tilde{\mathcal{H}}_{i}^{\prime}\left(\tilde{x}_{i}^{j-1}\right)\left(X_{i \mid i-1}^{j, n}-\tilde{x}_{i}^{j-1}\right)\right\|_{2 p}, \\
\leq & \left\|X_{0: i \mid i-1}^{j, n}\right\|_{p}+\left\|P_{0: i \mid i-1}^{j, N_{j}}\right\|_{8 p}\left\|\tilde{\mathcal{H}}_{i}^{\prime \mathrm{T}}\left(\tilde{x}_{i}^{j-1}\right)\right\|_{8 p} \\
& \cdot\left\|\left(\tilde{\mathcal{H}}_{i}^{\prime}\left(\tilde{x}_{i}^{j-1}\right) P_{0: i \mid i-1}^{j, N_{j}} \tilde{\mathcal{H}}_{i}^{\prime \mathrm{T}}\left(\tilde{x}_{i}^{j-1}\right)+\tilde{R}_{i}\right)^{-1}\right\|_{4 p} \\
& \cdot\left(\left|\tilde{y}_{i}\right|+\left\|\tilde{W}_{i}^{j, n}\right\|_{2 p}+\left\|\mathcal{H}_{i}\left(\tilde{x}_{i}^{j-1}\right)\right\|_{2 p}+\left\|\tilde{\mathcal{H}}_{i}^{\prime}\left(\tilde{x}_{i}^{j-1}\right)\right\|_{4 p}\left(\left\|X_{i \mid i-1}^{j, n}\right\|_{4 p}+\left\|\tilde{x}_{i}^{j-1}\right\|_{4 p}\right)\right) .
\end{aligned}
$$

Since $\tilde{R}_{i}$ is positive definite and $P_{0: i \mid i-1}^{j, N_{j}}$ is positive semi definite, we have

$$
\left\|\left(\tilde{\mathcal{H}}_{i}^{\prime}\left(\tilde{x}_{i}^{j-1}\right) P_{0: i \mid i-1}^{j, N_{j}} \tilde{\mathcal{H}}_{i}^{\prime \mathrm{T}}\left(\tilde{x}_{i}^{j-1}\right)+\tilde{R}_{i}\right)^{-1}\right\|_{4 p} \leq\left|\tilde{R}_{i}^{-1}\right|
$$

From [26, lemma 31] we have

$$
\left\|P_{0: i \mid i-1}^{j, N_{j}}\right\|_{8 p} \leq 2\left\|X_{0: i \mid i-1}^{j, 1}\right\|_{16 p}^{2}
$$

From the inequalities (39) and (40), Assumption 9, and the fact that $\tilde{W}_{i}^{j, n}$ is normally distributed, there exists a constant $\tilde{C}_{p}$ such that

$$
\begin{aligned}
\left\|X_{0: i \mid i}^{j, n}\right\|_{p} \leq & \left\|X_{0: i \mid i-1}^{j, n}\right\|_{p}+2\left\|X_{0: i \mid i-1}^{j, 1}\right\|_{16 p}^{2} \kappa \tilde{C}_{p}\left(1+\left\|\tilde{x}_{i}^{j-1}\right\|_{8 p s}^{s}\right) \\
& \left|\tilde{R}_{i}^{-1}\right|\left|\tilde{y}_{i}\right|+\tilde{C}_{p}+\kappa \tilde{C}_{p}\left(1+\left\|\tilde{x}_{i}^{j-1}\right\|_{2 p s}^{s}\right) \\
& +\kappa \tilde{C}_{p}\left(1+\left\|\tilde{x}_{i}^{j-1}\right\|_{4 p s}^{s}\right)\left(\left\|X_{i \mid i-1}^{j, 1}\right\|_{4 p}+\left\|\tilde{x}_{i}^{j-1}\right\|_{4 p}\right)
\end{aligned}
$$


Bounding $\tilde{x}_{i}^{j-1}$ by the induction assumption on $j$ and $X_{i-1 \mid i-1}^{j, n}$ by the induction assumption on $i$, we obtain that $\left\|X_{0: i \mid i}^{j, n, N_{j}}\right\|_{p}$ is bounded independently of $n$ and $N_{j}$.

At each iteration $j$ of Algorithm 8, we define for theoretical purposes a reference ensemble $\left[U_{0: k \mid k}^{1}, \ldots, U_{0: k \mid k}^{N_{j}}\right]$, similarly as in Section 5.3, and with the derivatives taken at the mean, rather than sample mean as in Algorithm 8: For $j=0$, all $U_{i \mid i}^{j, n}=X_{i \mid i}^{j, n}=x^{0}$ are constants. For $j=1,2, \ldots$, $U_{0 \mid 0}^{j, n}=X_{0 \mid 0}^{j, n}$ for $i=0$, and for $i=1, \ldots, k$,

$$
\begin{aligned}
U_{i \mid i-1}^{j, n} & =\mathcal{M}_{i}^{\prime}\left(E\left(U_{i-1 \mid i-1}^{j-1,1}\right)\right)\left(U_{i-1 \mid i-1}^{j, n}-x_{i-1}^{j-1}\right)+\mathcal{M}_{i}^{\prime}\left(E\left(U_{i-1}^{j-1,1}\right)\right)+\mu_{i}+V_{i}^{j, n} \\
U_{0: i \mid i}^{j, n} & =U_{0: i \mid i-1}^{j, n}+Q_{0: i}^{j} \tilde{\mathcal{H}}_{i}^{\prime}\left(E\left(U_{i \mid k}^{j-1,1}\right)\right)^{\mathrm{T}}\left(\mathcal{H}_{i}^{\prime}\left(E\left(U_{i \mid k}^{j-1,1}\right)\right) Q_{i}^{j} \mathcal{H}_{i}^{\prime}\left(E\left(U_{i \mid k}^{j-1,1}\right)\right)^{\mathrm{T}}+\tilde{R}_{i}\right)^{-1} \\
& \left(\hat{y}_{i}+\tilde{\mathcal{H}}_{i}^{\prime}\left(E\left(U_{i \mid k}^{j-1,1}\right)\right)\left(E\left(U_{i \mid k}^{j-1,1}\right)-U_{i \mid i-1}^{j, 1}\right)-\mathcal{H}_{i}\left(E\left(U_{i \mid i}^{j-1,1}\right)\right)-\tilde{W}_{i}^{j, 1}\right)
\end{aligned}
$$

where $Q_{0: i}^{j}=\operatorname{Cov}\left(U_{0: i \mid i-1}^{j-1,1}\right)$ and $\hat{y}_{i}=\left[\begin{array}{c}y_{i} \\ E\left(U_{i \mid k}^{j-1,1}\right)\end{array}\right]$. We now show that the mean of the reference ensemble members is the solution of the linearized least squares (27), and thus the next LM iterate:

Lemma 11. $E\left(U_{0: k \mid k}^{j, 1}\right)=x^{j}$, where $x^{j}$ is the $j$-th iterate generated by the algorithm (6).

Proof. The proof proceeds by induction on the iteration number $j$. For $j=0$ we have $U^{0, n}=x^{0}$ for all $n$ by definition, thus $E\left(U^{0,1}\right)=x^{0}$. Let $j \geq 1$. From the induction assumption, the stochastic system (41)-(42) is linearized about the previous LM iterate $x^{j-1}$. By Lemma $8,\left[U_{0: k}^{j, n}\right]_{n=1}^{N_{j}}$ are i.i.d. with the smoothing distribution, whose mean is the solution of the linearlized least squares (27).

Lemma 12. For all iterations $j$ and all times $i=0, \ldots, k$, the columns of the random matrix

$$
\left[X_{0: i \mid i}^{j} ; U_{0: i \mid i}^{j}\right]=\left[\begin{array}{c}
X_{0: i \mid i}^{j, 1}, \ldots, X_{0: i \mid i}^{j, N_{j}} \\
U_{0: i \mid i}^{j, 1}, \ldots, U_{0: i \mid i}^{j, N}
\end{array}\right]
$$

are exchangeable.

Proof. We recall that for all $j \geq 1, \tilde{x}^{j}=\bar{X}_{0: k \mid k}^{j, N_{j}}$. In this proof we omit the subscripts of $N_{j}$ and $N_{j-1}$. We use induction on the LM iteration number $j$. For $j=0$ we have that for all $i=0, \ldots, k$, $X_{0: i \mid i}^{0, n}=x_{0: i}^{0}=U_{0: i \mid i}^{0, n}$, and $U_{0: i \mid i}^{0, n}$ are i.i.d., therefore $\left[X_{0: i \mid i}^{0} ; U_{0: i \mid i}^{0}\right]$ are exchangeable. For $j \geq 1$, we use the induction on the time index $i$. For $i=0,\left[U_{0 \mid 0}^{j, n}\right]_{n=1}^{N}$ are i.i.d, and $X_{0 \mid 0}^{j, n}=U_{0 \mid 0}^{j, n}$, therefore 
$\left[X_{0 \mid 0}^{j} ; U_{0 \mid 0}^{j}\right]$ are exchangeable. For $i=1, \ldots, k$, consider first the forecast step,

$$
\begin{aligned}
{\left[\begin{array}{c}
X_{i \mid i-1}^{j, n} \\
U_{i \mid i-1}^{j, n}
\end{array}\right] } & =\left[\begin{array}{cc}
\mathcal{M}_{i}^{\prime}\left(\bar{X}_{i-1 \mid k}^{j-1, N}\right) & 0 \\
0 & \mathcal{M}_{i}^{\prime}\left(E\left(U_{i-1 \mid k}^{j-1,1}\right)\right)
\end{array}\right]\left[\begin{array}{c}
X_{i-1 \mid i-1}^{j, n} \\
U_{i-1 \mid i-1}^{j, n}
\end{array}\right] \\
+ & {\left[\begin{array}{c}
\mathcal{M}_{i}\left(\bar{X}_{i-1 \mid k}^{j-1, N}\right)-\mathcal{M}_{i}^{\prime}\left(\bar{X}_{i-1 \mid k}^{j-1, N}\right) \bar{X}_{i-1 \mid k}^{j-1, N}+\mu_{i} \\
\mathcal{M}_{i}\left(E\left(U_{i-1 \mid k}^{j-1}\right)\right)-\mathcal{M}_{i}^{\prime}\left(E\left(U_{i-1 \mid k}^{j-1,1}\right)\right) E\left(U_{i-1 \mid k}^{j-1,1}\right)+\mu_{i}
\end{array}\right]+\left[\begin{array}{c}
V_{i}^{j, n} \\
V_{i}^{j, n}
\end{array}\right] } \\
& =F^{i}\left(\bar{X}_{i-1 \mid k}^{j-1, N},\left[\begin{array}{c}
X_{i-1 \mid i-1}^{j, n} \\
U_{i-1 \mid i-1}^{j, n}
\end{array}\right],\left[\begin{array}{c}
V_{i}^{j, n} \\
V_{i}^{j, n}
\end{array}\right]\right),
\end{aligned}
$$

where $F^{i}$ is a measurable function. The ensemble sample mean $\bar{X}_{i-1 \mid k}^{j-1, N}$ is invariant to a permutation of ensemble members, and $V_{i}^{j}=\left[V_{i}^{j, 1}, \ldots, V_{i}^{j, N}\right]$ is exchangeable because its members are i.i.d. From the induction assumption on $i$ we have that $\left[X_{i-1 \mid i-1}^{j} ; U_{i-1 \mid i-1}^{j}\right]$ is exchangeable, and it is also independent from $\left[\begin{array}{c}V_{i}^{j} \\ V_{i}^{j}\end{array}\right]$, therefore, using Lemma $2,\left[\begin{array}{c}X_{i \mid i-1}^{j} \\ U_{i \mid i-1}^{j}\end{array}\right]$ is exchangeable. The analysis step also preserves exchageability:

$$
\begin{aligned}
{\left[\begin{array}{c}
X_{0: i \mid i}^{j, n} \\
U_{0: i \mid i}^{j, n}
\end{array}\right] } & =\left[\begin{array}{c}
X_{0: i \mid i-1}^{j, n} \\
U_{0: i \mid i-1}^{j, n}
\end{array}\right]+\left[\begin{array}{cc}
K_{i}^{N} & 0 \\
0 & K_{i}
\end{array}\right] \\
& \left(\left[\begin{array}{c}
\tilde{y}_{i}-\mathcal{H}_{i}^{\prime}\left(\bar{X}_{i \mid k}^{j-1, N}\right) \bar{X}_{i \mid i}^{j-1, N}-\mathcal{H}_{i}\left(\bar{X}_{i \mid k}^{j-1, N}\right)-\tilde{W}_{i}^{j, n} \\
\hat{y}_{i}-\mathcal{H}_{i}^{\prime}\left(E\left(U_{i \mid k}^{j-1,1}\right)\right) E\left(U_{i \mid k}^{j-1,1}\right)-\mathcal{H}_{i}\left(E\left(U_{i \mid k}^{j-1,1}\right)\right)-\tilde{W}_{i}^{j, n}
\end{array}\right]\right. \\
& \left.-\left[\begin{array}{cc}
\mathcal{H}_{i}^{\prime}\left(\bar{X}_{i \mid k}^{j-1, N}\right) & 0 \\
0 & \mathcal{H}_{i}^{\prime}\left(E\left(U_{i \mid k}^{j-1,1}\right)\right)
\end{array}\right]\left[\begin{array}{c}
X_{i \mid i-1}^{j, n} \\
U_{i \mid i-1}^{j, n}
\end{array}\right]\right) \\
& =F^{i}\left(\bar{X}_{i \mid k}^{j-1, N}, P_{0: i \mid i-1}^{j, N},\left[\begin{array}{c}
X_{i \mid i-1}^{j, n} \\
U_{i \mid i-1}^{j, n}
\end{array}\right],\left[\begin{array}{c}
\tilde{W}_{i}^{j, n} \\
\tilde{W}_{i}^{j, n}
\end{array}\right]\right)
\end{aligned}
$$

because the Kalman gain matrices are functions of the ensemble members through $\bar{X}_{i \mid k}^{j-1, N}$ and $P_{0: i \mid i-1}^{j, N}$ only,

$$
\begin{aligned}
K_{i}^{N}= & {\left[\begin{array}{c}
P_{0 \mid i-1}^{j, N} \mathcal{H}_{i}^{\prime}\left(\bar{X}_{i \mid k}^{j-1, N}\right)^{\mathrm{T}} \\
\vdots \\
P_{i \mid i-1}^{j, N} \mathcal{H}_{i}^{\prime}\left(\bar{X}_{i \mid k}^{j-1, N}\right)^{\mathrm{T}}
\end{array}\right]\left(\mathcal{H}_{i}^{\prime}\left(\bar{X}_{i \mid k}^{j-1, N j}\right) P_{i \mid i-1}^{j, N} \mathcal{H}_{i}^{\prime}\left(\bar{X}_{i \mid k}^{j-1, N}\right)^{\mathrm{T}}+\tilde{R}_{i}\right)^{-1}, } \\
K_{i}= & {\left[\begin{array}{c}
Q_{0}^{j} \mathcal{H}_{i}^{\prime}\left(E\left(U_{i \mid k}^{j-1,1}\right)\right)^{\mathrm{T}} \\
\vdots \\
Q_{i}^{j} \mathcal{H}_{i}^{\prime}\left(E\left(U_{i \mid k}^{j-1,1}\right)\right)^{\mathrm{T}}
\end{array}\right]\left(\mathcal{H}_{i}^{\prime}\left(E\left(U_{i \mid k}^{j-1,1}\right)\right) Q_{i}^{j} \mathcal{H}_{i}^{\prime}\left(E\left(U_{i \mid k}^{j-1,1}\right)\right)^{\mathrm{T}}+\tilde{R}_{i}\right)^{-1} }
\end{aligned}
$$


and the ensemble sample mean $\bar{X}_{i \mid k}^{j-1, N}$, and the ensemble sample covariance $P_{0: i \mid i-1}^{j, N}$ are invariant to a permutation of ensemble members, $\tilde{W}_{i}^{j}=\left[\tilde{W}_{i}^{j, 1}, \ldots, \tilde{W}_{i}^{j, N}\right]$ is exchangeable because, its members are i.i.d. and they are independent from $\left[X_{i \mid i-1}^{j, n} ; U_{i \mid i-1}^{j, n}\right]$ is exchangeable. Therefore, using again Lemma $2,\left[X_{0: i \mid i}^{j, n} ; U_{0: i \mid i}^{j, n}\right]$ are exchangeable.

Theorem 10. For all $j$, and $n=1, \ldots N_{j}, X_{0: k \mid k}^{j, n, N_{j}} \rightarrow U_{0: k \mid k}^{j, n}$ and $\bar{X}_{0: k \mid k}^{j, N_{j}} \rightarrow E\left(U_{0: k \mid k}^{j, 1}\right)$ as $\min \left\{N_{1}, \ldots, N_{j}\right\} \rightarrow$ $\infty$, in all $L^{p}, 1 \leq p<\infty$.

Proof. We will prove that for all $j \geq 0$ and all $1 \leq i \leq k, X_{0: i \mid i}^{j, 1, N_{j}} \rightarrow U_{0: i \mid i}^{j, 1}$ as $\min \left\{N_{1}, \ldots, N_{j}\right\} \rightarrow \infty$, in all $L^{p}, 1 \leq p<\infty$, and the convergence of the mean follows. Since $\left[X_{0: i \mid i}^{j, n} ; U_{0: i \mid i}^{j, n}\right]_{n=1}^{N_{j}}$ are exchangeable, we only need to consider the convergence of $X_{0: i \mid i}^{j, 1, N_{j}} \rightarrow U_{0: i \mid i}^{j, 1}$. We use induction on the LM iteration number $j$. For $j=0$, we have $X_{0: i \mid i}^{0,1, N_{0}}=x_{0: i}^{0}=U_{0: i \mid i}^{0,1}$. For $j \geq 1$, we use induction on time index $i$. For $i=0, X_{0 \mid 0}^{j, 1, N_{j}}=U_{0 \mid 0}^{j, 1}$. For $i=1, \ldots, k$, from induction assumption on $j$ and $i$, we have $\bar{X}_{i-1 \mid k}^{j-1, N_{j}} \rightarrow E\left(U_{i-1 \mid k}^{j-1,1}\right)$ and $X_{i-1 \mid k}^{j, 1, N_{j}} \rightarrow U_{i-1 \mid k}^{j, 1}$ in all $L^{p}$, $1 \leq p<\infty$, as $\min \left\{N_{1}, \ldots, N_{j}\right\} \rightarrow \infty$. Convergence in $L^{p}$ implies convergence in probability, and by the continuous mapping theorem,

$$
\begin{aligned}
X_{i \mid i-1}^{j, 1, N_{j}}= & \mathcal{M}_{i}^{\prime}\left(\bar{X}_{i-1 \mid k}^{j-1, N_{j-1}}\right) X_{i-1 \mid i-1}^{j, 1, N_{j}}+\mathcal{M}_{i}\left(\bar{X}_{i-1 \mid k}^{j-1, N_{j-1}}\right) \\
& -\mathcal{M}_{i}^{\prime}\left(\bar{X}_{i-1 \mid k}^{j-1, N_{j-1}}\right) \bar{X}_{i-1 \mid k}^{j-1, N_{j-1}}+\mu_{i}+V_{i}^{j, 1} \\
\stackrel{\mathrm{P}}{\rightarrow} & \mathcal{M}_{i}^{\prime}\left(E\left(U_{i-1 \mid k}^{j-1,1}\right)\right) U_{i-1 \mid i-1}^{j, 1}+\mathcal{M}_{i}\left(E\left(U_{i-1 \mid k}^{j-1,1}\right)\right) \\
& -\mathcal{M}_{i}^{\prime}\left(E\left(U_{i-1 \mid k}^{j-1,1}\right)\right) E\left(U_{i-1 \mid k}^{j-1,1}\right)+\mu_{i}+V_{i}^{j, 1} \\
= & U_{i \mid i-1}^{j, 1}
\end{aligned}
$$

as $\min \left\{N_{1}, \ldots, N_{j}\right\} \rightarrow \infty$. From Lemma 10, the sequence $\left\{X_{0: i \mid i-1}^{j, 1, N_{j}}\right\}_{N_{j}=1}^{\infty}$ is bounded in all $L^{p}$, $1 \leq p<\infty$, therefore by using the uniform integrability theorem we leverage the convergence in probability to convergence in all $L^{p}$, hence $X_{0: i \mid i-1}^{j, 1, N_{j}} \rightarrow U_{0: i \mid i-1}^{j, 1}$ and $\bar{X}_{0: i \mid i-1}^{j, N_{j}} \rightarrow E\left(U_{0: i \mid i-1}^{j, 1}\right)$ in all $L^{p}$. From [26] we have $P_{0: i \mid i-1}^{j, N_{j}} \stackrel{\mathrm{P}}{\rightarrow} Q_{0: i}^{j}$, then, from the continuous mapping theorem, $K_{i}^{N_{j}} \stackrel{\mathrm{P}}{\rightarrow} K_{i}$. From the fact that convergence in $L^{p}$ implies convergence in probability, and using the continuous mapping theorem again, we conclude that

$$
\begin{aligned}
X_{0: i \mid i}^{j, 1, N_{j}}= & X_{0: i \mid i-1}^{j, 1, N_{j}}+K_{i}^{N_{j}}\left(\tilde{y}_{i}+\tilde{\mathcal{H}}_{i}^{\prime}\left(\bar{X}_{i \mid k}^{j-1, N_{j-1}}\right) \bar{X}_{i \mid k}^{j-1, N_{j-1}}-\tilde{\mathcal{H}}_{i}\left(\bar{X}_{i \mid k}^{j-1, N_{j-1}}\right)\right. \\
& \left.-\tilde{W}_{i}^{j, 1}-\tilde{\mathcal{H}}_{i}^{\prime}\left(\bar{X}_{i \mid k}^{j-1, N_{j-1}}\right) X_{i \mid i-1}^{j, 1, N_{j}}\right) \\
\stackrel{\mathrm{P}}{\rightarrow} & U_{0: i \mid i-1}^{j, 1}+K_{i}\left(\hat{y}_{i}+\tilde{\mathcal{H}}_{i}^{\prime}\left(E\left(U_{i \mid k}^{j-1,1}\right)\right) E\left(U_{i \mid k}^{j-1,1}\right)-\tilde{\mathcal{H}}_{i}\left(E\left(U_{i \mid i}^{j-1,1}\right)\right)\right. \\
& \left.-\tilde{W}_{i}^{j, 1}-\tilde{\mathcal{H}}_{i}^{\prime}\left(E\left(U_{i \mid k}^{j-1,1}\right)\right) U_{i \mid i-1}^{j, 1}\right) \\
= & U_{0: i \mid i}^{j, 1},
\end{aligned}
$$

as $\min \left\{N_{1}, \ldots, N_{j}\right\} \rightarrow \infty$. Then we leverage the last convergence to the convergence in $L^{p}$ using Lemma 10 and the uniform integrability again. 


\subsection{EnKS-4DVAR}

To avoid computing with the tangent matrices $\mathcal{M}_{i}^{\prime}\left(x_{i-1}^{j-1}\right)$ and

$\mathcal{H}_{i}^{\prime}\left(x_{i}^{j-1}\right)$, we take advantage of the fact that they occur in the EnKS only in matrix-vector products, and approximate the matrix-vector multiplications in Algorithm 8 by finite differences with a small step size $\tau>0$, centered at the previous iterate. Thus, we use the approximations of the form

$$
f^{\prime}(x) y \approx \frac{f(x+\tau y)-f(y)}{\tau}
$$

in (35), (36), and (37). Denote by an additional superscript $\tau$ the quantities computed in the resulting algorithm. This is the EnKS-4DVAR method originally proposed in [25].

Algorithm 11 (EnKS-4DVAR). Given an initial approximation $x_{0: k}^{0}, \gamma>0$, and $\tau>0$. Initialize

$$
\bar{X}_{0: k \mid k}^{j, N_{j}, \tau}=x_{0: k}^{0} \quad \text { for } j=0 .
$$

LM loop: For $j=1,2, \ldots$ Choose $N_{j}$ the same as in Algorithm 8.

EnKS loop: For $i=0$, the ensemble $\left[X_{0 \mid 0}^{j, n, \tau}\right]_{n=1}^{N_{j}}$ consists of i.i.d. Gaussian random variables

$$
X_{0 \mid 0}^{j, n, \tau} \sim N\left(\tilde{x}_{0}^{0}, B\right)
$$

For $i=1, \ldots, k$, advance the model in time (the forecast step) by

$$
\begin{aligned}
X_{i \mid i-1}^{j, n, \tau} & =\frac{\mathcal{M}_{i}\left(\bar{X}_{i-1 \mid k}^{j-1, N_{j-1}, \tau}+\tau\left(X_{i-1 \mid i-1}^{j, n, \tau}-\bar{X}_{i-1 \mid k}^{j-1, N_{j-1}, \tau}\right)\right)-\mathcal{M}_{i}\left(\bar{X}_{i-1 \mid k}^{j-1, N_{j-1}, \tau}\right)}{\tau}, \\
& +\mathcal{M}_{i}\left(\bar{X}_{i-1 \mid k}^{j-1, N_{j-1}, \tau}\right)+\mu_{i}+V_{i}^{j, n} \quad V_{i}^{j, n} \sim N\left(0, Q_{i}\right), \quad n=1, \ldots, N_{j} .
\end{aligned}
$$

Incorporate the observations at time $i$ into the ensemble of composite states $\left[X_{0: i \mid i-1}^{j, n, \tau}\right]_{n=1}^{N_{j}}$ by the analysis step

$$
\begin{aligned}
X_{0: i \mid i}^{j, n, \tau}= & X_{0: i \mid i-1}^{j, n, \tau}+P_{0: i \mid i-1}^{j, N_{j}, \tau} \tilde{H}_{i}^{j, \tau \mathrm{T}}\left(\tilde{H}_{i}^{j, \tau} P_{0: i \mid i-1}^{j, N_{j}, \tau} \tilde{H}_{i}^{j, \tau \mathrm{T}}+\tilde{R}_{i}\right)^{-1} \\
& \cdot\left(\tilde{y}_{i}-\tilde{W}_{i}^{j, n}-\tilde{\mathcal{H}}_{i}\left(\bar{X}_{i \mid k}^{j-1, N_{j-1}, \tau}\right)\right. \\
& \left.\quad-\frac{\tilde{\mathcal{H}}_{i}\left(\bar{X}_{i \mid k}^{j-1, N_{j-1}, \tau}+\tau\left(X_{i \mid i-1}^{j, n, \tau}-\bar{X}_{i \mid k}^{j-1, N_{j-1}, \tau}\right)\right)-\tilde{\mathcal{H}}_{i}\left(\bar{X}_{i \mid k}^{j-1, N_{j-1}, \tau}\right)}{\tau}\right), \\
& \quad \tilde{W}_{i}^{j, n} \sim N\left(0, \tilde{R}_{i}\right)
\end{aligned}
$$

where $P_{0: i \mid i-1}^{j, N_{j}, \tau}$ is the sample covariance from the ensemble $\left[X_{0: i \mid i-1}^{j, n, \tau}\right]_{n=1}^{N_{j}}$. Similarly as in (9)-(11), 
only the following matrix-vector products are needed:

$$
\begin{aligned}
P_{0: i \mid i-1}^{j, N_{j}, \tau} \tilde{H}_{i}^{j, \tau \mathrm{T}} & =\frac{1}{N_{j}-1} \sum_{n=1}^{N_{j}}\left(X_{0: i \mid i-1}^{j, n, \tau}-\bar{X}_{0: i \mid i-1}^{j, N_{j}, \tau}\right)\left(X_{i \mid i-1}^{j, n, \tau}-\bar{X}_{i \mid i-1}^{j, N_{j}, \tau}\right)^{\mathrm{T}} \tilde{H}_{i}^{j, \tau \mathrm{T}}, \\
& =\frac{1}{N_{j}-1} \sum_{n=1}^{N_{j}}\left(X_{0: i \mid i-1}^{j, n, \tau}-\bar{X}_{0: i \mid i-1}^{j, N_{j}, \tau}\right) h_{i}^{j, n, \tau \mathrm{T}} \\
\tilde{H}_{i}^{j} P_{0: i \mid i-1}^{j, N_{j}, \tau} \tilde{H}_{i}^{j, \tau \mathrm{T}} & =\frac{1}{N_{j}-1} \sum_{n=1}^{N_{j}} \tilde{H}_{i}^{j, \tau}\left(X_{i \mid i-1}^{j, n, \tau}-\bar{X}_{i \mid i-1}^{j, N_{j}, \tau}\right)\left(X_{i \mid i-1}^{j, n, \tau}-\bar{X}_{i \mid i-1}^{j, N_{j}, \tau}\right)^{\mathrm{T}} \tilde{H}_{i}^{j, \tau \mathrm{T}} \\
& =\frac{1}{N_{j}-1} \sum_{n=1}^{N_{j}} h_{i}^{j, n, \tau} h_{i}^{j, n, \tau \mathrm{T}},
\end{aligned}
$$

where

$$
h_{i}^{j, n, \tau}=\tilde{H}_{i}^{j, \tau}\left(X_{i \mid i-1}^{j, n, \tau}-\bar{X}_{i \mid i-1}^{j, N_{j}, \tau}\right)=\frac{\tilde{\mathcal{H}}_{i}\left(\tau\left(X_{i \mid i-1}^{j, n, \tau}-\bar{X}_{i \mid i-1}^{j, N_{j}, \tau}\right)+\bar{X}_{i \mid k}^{j-1, N_{j-1}, \tau}\right)-\tilde{\mathcal{H}}_{i}\left(\bar{X}_{i \mid k}^{j-1, N_{j-1}, \tau}\right)}{\tau}
$$

and

$$
\bar{X}_{i \mid i-1}^{j, N_{j}, \tau}=\frac{1}{N_{j}} \sum_{n=1}^{N_{j}} X_{i \mid i-1}^{j, n, \tau}, \quad \bar{X}_{0: i \mid i}^{j, N_{j}, \tau}=\frac{1}{N_{j}} \sum_{n=1}^{N_{j}} X_{0: i \mid i}^{j, n, \tau} .
$$

The next LM iterate is $\tilde{x}^{j, \tau}=\bar{X}_{0: k \mid k}^{j, N_{j}, \tau}$.

We now summarize the differences between the previous three algorithms. Algorithm 6 solves the linearized problem in each iteration exactly, while Algorithm 8 approximates the solution of the linearized problem by EnKS, and Algorithm 11 approximates also the linearized problem itself by finite differences.

We show that when the finite difference parameter $\tau \rightarrow 0$, the iterations of Algorithm 11 converge to their corresponding iterations of Algorithm 8 in probability. The following lemma is the cornerstone of the analysis of the finite differences here.

Lemma 13. Let $\left(X_{\tau}\right)$ and $\left(Y_{\tau}\right)$ be random vectors such that $X_{\tau} \stackrel{\mathrm{P}}{\rightarrow} X$ and $Y_{\tau} \stackrel{\mathrm{P}}{\rightarrow} Y$ as $\tau \rightarrow 0$, $\tau>0$, and $f$ be twice continuously differentiable with the matrix of second order derivatives $f^{\prime \prime}$ bounded. Then,

$$
\frac{f\left(X_{\tau}+\tau Y_{\tau}\right)-f\left(X_{\tau}\right)}{\tau} \stackrel{\mathrm{P}}{\rightarrow} f^{\prime}(X) Y \text { as } \tau \rightarrow 0, \tau>0
$$

Proof. From Taylor expansion, for any $x, y$, and $t$,

$$
\left|\frac{f(x+t y)-f(x)}{t}-f^{\prime}(x) y\right| \leq M t|y|^{2},
$$

where $M=\frac{1}{2} \sup _{\xi}\left|f^{\prime \prime}(\xi)\right|$ in the matrix norm induced by the vector norm $|\cdot|$. Let $\varepsilon>0, \tilde{\varepsilon}>0$. Since $Y_{\tau} \stackrel{\mathrm{P}}{\rightarrow} Y,\left\{Y_{\tau}\right\}$ is uniformly tight, that is, there exists $K$ such that $\mathbb{P}\left[\left|Y_{\tau}\right| \leq K\right] \geq 1-\tilde{\varepsilon}$ for all $\tau>0$. Choose $\tau_{1}=\frac{\varepsilon}{M K^{2}}>0$. Using (50), it follows that for all $0<\tau<\tau_{1}$,

$$
\mathbb{P}\left[\left|\frac{f\left(X_{\tau}+\tau Y_{\tau}\right)-f\left(X_{\tau}\right)}{\tau}-f^{\prime}\left(X_{\tau}\right) Y_{\tau}\right| \leq \varepsilon\right] \geq 1-\tilde{\varepsilon} .
$$


Since the mapping $(x, y) \mapsto f^{\prime}(x) y$ is continuous and $\left(X_{\tau}, Y_{\tau}\right) \rightarrow(X, Y)$ in probability, it follows from the continuous mapping theorem that $f^{\prime}\left(X_{\tau}\right) Y_{\tau} \rightarrow f^{\prime}(X) Y$ in probability, hence there exists $\tau_{2}$ such that for all $\tau<\tau_{2}$,

$$
\mathbb{P}\left[\left|f^{\prime}\left(X_{\tau}\right) Y_{\tau}-f^{\prime}(X) Y\right| \leq \varepsilon\right] \geq 1-\tilde{\varepsilon} .
$$

Finally, using the triangle inequality, (51) and (52) imply

$$
\mathbb{P}\left[\left|\frac{f\left(X_{\tau}+\tau Y_{\tau}\right)-f\left(X_{\tau}\right)}{\tau}-f^{\prime}(X) Y\right| \leq 2 \varepsilon\right] \geq 1-2 \tilde{\varepsilon},
$$

for all $0<\tau<\min \left\{\tau_{1}, \tau_{2}\right\}$.

Theorem 12. At each iteration $j$ and time step $i$ of Algorithm $11, X_{0: i \mid i}^{j, n, \tau} \stackrel{\mathrm{P}}{\rightarrow} X_{0: i \mid i}^{j, n}$ as $\tau \rightarrow 0$, where $X_{0: i \mid i}^{j, n}$ is the $n$-th member of the ensemble generated at $j$-th iteration in Algorithm 8 with the same random perturbations as in Algorithm 11.

Proof. In this proof we omit the subscripts of $N_{j}$ and $N_{j-1}$. The proof is by induction on the number of iterations $j$. For $j=1$ we have $\bar{X}_{0: i \mid i}^{j-1, N, \tau}=\bar{X}_{0: i \mid i}^{j-1, N}$. For $j \geq 2$, we use induction on time step $i$. For $i=0$ we have $X_{0 \mid 0}^{j, n, \tau}=x_{\mathrm{b}}+V_{b}^{n}=X_{0 \mid 0}^{j, n}$. For $i=1, \ldots, k$, we have from the induction assumption on $i, X_{i-1 \mid i-1}^{j, n, \tau} \stackrel{\mathrm{P}}{\rightarrow} X_{i-1 \mid i-1}^{j, n}$ as $\tau \rightarrow 0$. Then using Lemma 13, we have in (45) as $\tau \rightarrow 0$,

$$
\begin{aligned}
X_{i \mid i-1}^{j, n, \tau}= & \frac{\mathcal{M}_{i}\left(\bar{X}_{i-1 \mid k}^{j-1, N, \tau}+\tau\left(X_{i-1 \mid i-1}^{j, n, \tau}-\bar{X}_{i-1 \mid k}^{j-1, N, \tau}\right)\right)-\mathcal{M}_{i}\left(\bar{X}_{i-1 \mid k}^{j-1, N, \tau}\right)}{\tau} \\
& +\mathcal{M}_{i}\left(\bar{X}_{i-1 \mid k}^{j-1, N, \tau}\right)+\mu_{i} \\
\stackrel{\mathrm{P}}{\rightarrow} & \mathcal{M}_{i}^{\prime}\left(\bar{X}_{i-1 \mid k}^{j-1, N}\right)\left(X_{i-1 \mid i-1}^{j, n}-\bar{X}_{i-1 \mid k}^{j-1, N}\right)+\mathcal{M}_{i}\left(\bar{X}_{i-1}^{j-1, N}\right)+\mu_{i}+V_{i}^{n}=X_{i \mid i-1}^{j, n} .
\end{aligned}
$$

Similarly, using the induction assumption on $j$ and Lemma 13, we have in (46) and in (49), respectively,

$$
\begin{aligned}
& \frac{\mathcal{H}_{i}\left(\bar{X}_{i \mid k}^{j-1, N, \tau}+\tau\left(X_{i \mid i-1}^{j, n, \tau}-\bar{X}_{i \mid k}^{j-1, N, \tau}\right)\right)-\mathcal{H}_{i}\left(\bar{X}_{i \mid k}^{j-1, N, \tau}\right)}{\tau} \\
& \stackrel{\stackrel{\mathrm{P}}{\rightarrow} \mathcal{H}_{i}^{\prime}\left(\bar{X}_{i \mid i}^{j-1, N}\right)\left(X_{i \mid i-1}^{j, n, \tau}-\bar{X}_{i \mid k}^{j-1, N, \tau}\right),}{\frac{\mathcal{H}_{i}\left(\bar{X}_{i \mid k}^{j-1, N, \tau}+\tau\left(X_{i \mid i-1}^{j, n, \tau}-\bar{X}_{i \mid i-1}^{j-1, N, \tau}\right)\right)-\mathcal{H}_{i}\left(\bar{X}_{i \mid k}^{j-1, N, \tau}\right)}{\tau}} \\
& \stackrel{\mathrm{P}}{\rightarrow} \mathcal{H}_{i}^{\prime}\left(\bar{X}_{i \mid k}^{j-1, N}\right)\left(X_{i \mid i-1}^{j, n, \tau}-\bar{X}_{i \mid-1}^{j-1, N, \tau}\right) .
\end{aligned}
$$

as $\tau \rightarrow 0$. In (11) gives

$$
h_{i}^{j, n, \tau} \stackrel{\mathrm{P}}{\rightarrow} h_{i}^{j, n} \text { as } \tau \rightarrow 0 .
$$

Using (55) and the continuous mapping theorem in (48) and (47) gives

$$
\begin{aligned}
P_{0: i \mid i-1}^{j, N, \tau} \tilde{H}_{i}^{j, \tau \mathrm{T}} \stackrel{\mathrm{P}}{\rightarrow} P_{0: i \mid i-1}^{j, N} \tilde{H}_{i}^{j \mathrm{~T}} \quad \text { as } \tau \rightarrow 0, \\
\tilde{H}_{i}^{j, \tau} P_{0: i \mid i-1}^{j, N, \tau} \tilde{H}_{i}^{j, \tau \mathrm{T}} \stackrel{\mathrm{P}}{\rightarrow} \tilde{H}_{i}^{j} P_{0: i \mid i-1}^{j, N} \tilde{H}_{i}^{j \mathrm{~T}} \quad \text { as } \tau \rightarrow 0 .
\end{aligned}
$$


Using also (53) in (46) and the continuous mapping theorem once more gives $X_{0: i \mid i}^{j, n} \stackrel{\mathrm{P}}{\rightarrow} X_{0: i \mid i}^{j, n}$ as $\tau \rightarrow 0$.

Corollary 13. For each $j, \lim _{\min \left\{N_{1}, \ldots, N_{j}\right\} \rightarrow \infty} \lim _{\tau \rightarrow 0} \bar{X}_{0: k \mid k}^{j, N, \tau}=x^{j}$ in probability, where $x^{j}$ is the $j$-th iterate of Algorithm 6.

Proof. The proof follows immediately from Theorem 12, Theorem 10, and Lemma 11.

\section{Conclusion}

In this paper we have shown that: when the observation and the model operators are linear for any time step, the empirical mean and covariance of EnKS converge to the KS mean and covariance in the limit for large ensemble size in $L^{p}$ for any $p \in[1, \infty)$. In the nonlinear case, i.e., in the case where the observation and the model operators are not necessary linear, we have shown the convergence of LM-EnKS iterations (Algorithm 11) in the limit for large ensemble size. The convergence is in the sense that (i) each iterate generated by Algorithm 11 converges in probability to its corresponding iterate of Algorithm 8 as the finite differences parameter goes to zero, (ii) and that each iterate generated by Algorithm 8 converges, in $L^{p}$ for any $p \in[1, \infty)$, to its corresponding iterate of Algorithm 6 (the Levenberg-Marquardt algorithm) in the large-ensemble limit.

These proofs of convergence, and more generally the asymptotic behavior of the ensemblebased algorithms deserve further investigation. Here in the nonlinear case, we have given only the limit in probability of each iterate of Algorithm 11 as the finite differences parameter goes to zero and the ensemble sizes go to infinity. One may, for instance, try to prove stronger convergence results, especially to leverage the convergences in probability to convergences in $L^{p}$, and show the convergence rate of these algorithms following the spirit of [19]. The approach followed in this paper could be also extended to the case in which other variants of ensemble method, such as the square root ensemble Kalman filter [17], are used to approximately solve the linearized subproblem.

\section{References}

[1] B. D. O. Anderson and J. B. Moore. Optimal filtering. Prentice-Hall, Englewood Cliffs, N.J., 1979 .

[2] B. M. Bell and F. W. Cathey. The iterated Kalman filter update as a Gauss-Newton method. IEEE Transactions on Automatic Control, 38(2):294-297, Feb 1993.

[3] P. Billingsley. Probability and measure. John Wiley \& Sons Inc., New York, third edition, 1995.

[4] M. Bocquet and P. Sakov. Combining inflation-free and iterative ensemble Kalman filters for strongly nonlinear systems. Nonlinear Processes in Geophysics, 19(3):383-399, 2012.

[5] M. Bocquet and P. Sakov. An iterative ensemble Kalman smoother. Quarterly Journal of the Royal Meteorological Society, 140:1521-1535, 2014.

[6] G. Burgers, P. J. van Leeuwen, and G. Evensen. Analysis scheme in the ensemble Kalman filter. Monthly Weather Review, 126:1719-1724, 1998. 
[7] P. Courtier, J. N. Thépaut, and A. Hollingsworth. A strategy for operational implementation of 4D-Var, using an incremental approach. Quarterly Journal of the Royal Meteorological Society, 120(519):1367-1387, 1994.

[8] G. Evensen. Data Assimilation: The Ensemble Kalman Filter. Springer, 2nd edition, 2009.

[9] M. Fisher, M. Leutbecher, and G. A. Kelly. On the equivalence between Kalman smoothing and weak-constraint four-dimensional variational data assimilation. Quarterly Journal of the Royal Meteorological Society, 131(613, Part c):3235-3246, OCT 2005.

[10] P. E. Gill and W. Murray. Algorithms for the solution of the nonlinear least-squares problem. SIAM J. Numer. Anal., 15(5):977-992, 1978.

[11] T. M. Hamill and C. Snyder. A hybrid ensemble Kalman filter-3D variational analysis scheme. Monthly Weather Review, 128(8):2905-2919, 2000.

[12] C. J. Johns and J. Mandel. A two-stage ensemble Kalman filter for smooth data assimilation. Environmental and Ecological Statistics, 15:101-110, 2008.

[13] R. E. Kalman. A new approach to linear filtering and prediction problems. Transactions of the ASME - Journal of Basic Engineering, Series D, 82:35-45, 1960.

[14] E. Kalnay. Ensemble Kalman filter: Current status and potential. In William Lahoz, Boris Khattatov, and Richard Ménard, editors, Data Assimilation, pages 69-92. Springer Berlin Heidelberg, 2010.

[15] D. T. B. Kelly, K. J. H. Law, and A. M. Stuart. Well-posedness and accuracy of the ensemble Kalman filter in discrete and continuous time. Nonlinearity, 27(10):2579-2603, 2014.

[16] S. P. Khare, J. L. Anderson, T. J. Hoar, and D. Nychka. An investigation into the application of an ensemble Kalman smoother to high-dimensional geophysical systems. Tellus A, 60(1):97$112,2008$.

[17] E. Kwiatkowski and J. Mandel. Convergence of the square root ensemble Kalman filter in the large ensemble limit. SIAM/ASA Journal on Uncertainty Quantification, 3(1):1-17, 2015.

[18] F. X. Le Dimet and O. Talagrand. Variational algorithms for analysis and assimilation of meteorological observations: theoretical aspects. Tellus A, 38:97-110, 1986.

[19] F. Le Gland, V. Monbet, and V. D. Tran. Large sample asymptotics for the ensemble Kalman filter. In Dan Crisan and Boris Rozovskiǐ, editors, The Oxford Handbook of Nonlinear Filtering, pages 598-631. Oxford University Press, 2011.

[20] K. Levenberg. A method for the solution of certain non-linear problems in least squares. Quarterly of Applied Mathematics, 2:164-168, 1944.

[21] Z. Li and I. M. Navon. Optimality of variational data assimilation and its relationship with the Kalman filter and smoother. Quarterly Journal of the Royal Meteorological Society, 127(572):661-683, 2001. 
[22] C. Liu and Q. Xiao. An ensemble-based four-dimensional variational data assimilation scheme. Part III: Antarctic applications with Advanced Research WRF using real data. Monthly Weather Review, 141(8):2721-2739, 2013.

[23] C. Liu, Q. Xiao, and B. Wang. An ensemble-based four-dimensional variational data assimilation scheme. Part I: Technical formulation and preliminary test. Monthly Weather Review, 136(9):3363-3373, 2008.

[24] C. Liu, Q. Xiao, and B. Wang. An ensemble-based four-dimensional variational data assimilation scheme. Part II: Observing system simulation experiments with Advanced Research WRF (ARW). Monthly Weather Review, 137(5):1687-1704, 2009.

[25] J. Mandel, E. Bergou, and S. Gratton. 4DVAR by ensemble Kalman smoother. arxiv:1304.5271, 2013.

[26] J. Mandel, L. Cobb, and J. D. Beezley. On the convergence of the ensemble Kalman filter. Applications of Mathematics, 56:533-541, 2011.

[27] D. W. Marquardt. An algorithm for least-squares estimation of nonlinear parameters. Journal of the Society for Industrial and Applied Mathematics, 11:431-441, 1963.

[28] M. R. Osborne. Nonlinear least squares - the Levenberg algorithm revisited. Journal of the Australian Mathematical Society Series B, 19(3):343-357, 1976.

[29] P. Sakov, D. S. Oliver, and L. Bertino. An iterative EnKF for strongly nonlinear systems. Monthly Weather Review, 140(6):1988-2004, 2012.

[30] D. Simon. Optimal State Estimation: Kalman, $\mathrm{H}_{\infty}$, and Nonlinear Approaches. John Wiley and Sons, 2006.

[31] Y. Trémolet. Model-error estimation in 4D-Var. Quarterly Journal of the Royal Meteorological Society, 133(626):1267-1280, 2007.

[32] J. Tshimanga, S. Gratton, A. T. Weaver, and A. Sartenaer. Limited-memory preconditioners, with application to incremental four-dimensional variational data assimilation. Quarterly Journal of the Royal Meteorological Society, 134(632):751-769, 2008.

[33] A. W. Van der Vaart. Asymptotic Statistics. Cambridge University Press, 2000.

[34] X. Wang. Incorporating ensemble covariance in the gridpoint statistical interpolation variational minimization: A mathematical framework. Monthly Weather Review, 138(7):2990-2995, 2010 .

[35] M. Zupanski. Maximum likelihood ensemble filter: Theoretical aspects. Monthly Weather Review, 133(6):1710-1726, 2013/01/01 2005. 